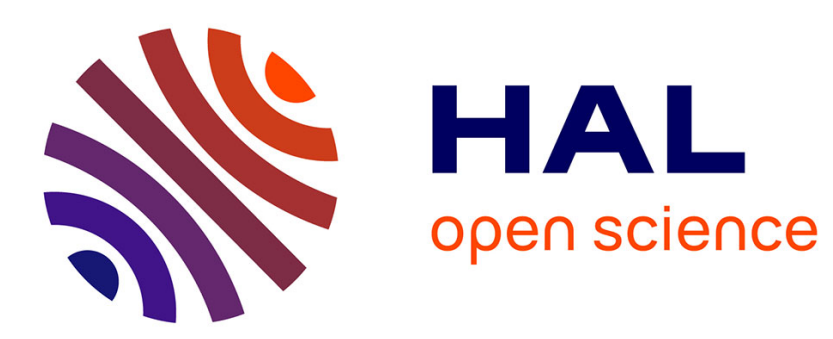

\title{
Static grounds for inequalities in finite strain of elastic materials
}

\author{
C. Truesdell, R. Toupin
}

\section{To cite this version:}

C. Truesdell, R. Toupin. Static grounds for inequalities in finite strain of elastic materials. Archive for Rational Mechanics and Analysis, 1963, 12 (1), pp.1-33. hal-00852708

\section{HAL Id: hal-00852708 \\ https://hal.science/hal-00852708}

Submitted on 27 Aug 2013

HAL is a multi-disciplinary open access archive for the deposit and dissemination of scientific research documents, whether they are published or not. The documents may come from teaching and research institutions in France or abroad, or from public or private research centers.
L'archive ouverte pluridisciplinaire HAL, est destinée au dépôt et à la diffusion de documents scientifiques de niveau recherche, publiés ou non, émanant des établissements d'enseignement et de recherche français ou étrangers, des laboratoires publics ou privés. 


\title{
Static Grounds for Inequalities in Finite Strain of Elastic Materials
}

\author{
C. TRUESDELL \& R. TOUPIN
}

\begin{abstract}
Contents
1. Nature and origin of the problem . . . . . . . . . . . . . . . . 1

2. List of formulae from the theory of elasticity . . . . . . . . . . . . . . . 4

3. Some plausible static inequalities for isotropic materials . . . . . . . . . . 7

4. Some logical connections among the static inequalities . . . . . . . . . . . 11

5. The Coleman-Noll and GCN conditions . . . . . . . . . . . . . . . . 18

6. The $\mathrm{C}_{-} \mathrm{N}^{+}$and $\mathrm{GCN}^{+}$conditions . . . . . . . . . . . . . . . . . . . . . 20

7. The condition of strong ellipticity . . . . . . . . . . . . . . . . . . . 24

8. Three uniqueness theorems . . . . . . . . . . . . . . . . . . . . . . . 26

9. General conclusions . . . . . . . . . . . . . . . . . . . 30

References ... . . . . . . . . . . . . . . . . . 31
\end{abstract}

\section{Nature and origin of the problem}

The two elasticities, $\lambda$ and $\mu$, occurring in the classical linearized theory of isotropic elastic materials are not entirely arbitrary constants or functions of temperature. In order that the equations describe a response that is physically reasonable, the inequalities

$$
\mu>0, \quad 3 \lambda+2 \mu>0,
$$

are usually imposed. These restrictions may be called the $C$-inequalities, where " $C$ " is a mnemonic for "classical". The problem of finding corresponding restrictions for finitely elastic materials was proposed in a lecture by TRUESDELL 1 in 1955, who summarized what was then known concerning it. As he remarked, the C-inequalities arise in connection with several different kinds of argument:

\section{A. Plausibility in statics.}

A1. In special strains. The condition (1.1) is necessary and sufficient that in simple shear, the shearing stress be directed in the same sense as the shear effected. The condition (1.1) $)_{2}$ is necessary and sufficient that tension be required to produce a uniform dilation, while pressure be required to produce a uniform condensation.

A2. In general strains. In any strain whatever, the condition $(1.1)_{1}$ is necessary and sufficient that the shearing stress on any plane shall have the same direc-

$1[1956,2]$. A survey of work done since 1955 on the group of problems related to the "Hauptproblem" was given by Toupin in a lecture to the British Theoretical Mechanics Colloquium at Newcastle-upon-Tyne in 1961. 
tion as the shear of that plane. The condition $(1.1)_{2}$ is necessary and sufficient that the mean tension have the same sign as the increment in volume.

$B$. Work theorem. The $\mathrm{C}$-inequalities are necessary and sufficient that the work done in any non-rigid deformation be positive.

C. Stability. The C-inequalities are sufficient but not necessary in order that the displacement satisfying the conditions of equilibrium shall correspond to a lesser total stored energy than does any other displacement having the same boundary values. Necessary and sufficient conditions are given by the weaker inequalities ${ }^{2}$

$$
\mu>0, \quad \lambda+2 \mu>0 .
$$

(It is important to distinguish between this condition and the preceding. In essence, the work theorem imposes the local requirement that the energy stored in every finite portion of the deformed material be positive, while the stability theorem is global, referring only to the total energy stored by a (necessarily non-equilibrated if non-vanishing) deformation within a fixed boundary.)

D. Uniqueness theorems. The $\mathrm{C}$-inequalities are sufficient in order that the mixed boundary-value problem of equilibrium, for smooth enough boundaries and boundary data, have a solution that is unique, at least to within a rigid motion. Moreover, the $\mathrm{C}$-inequalities or their opposites are also necessary in order that, for a general region, both the displacement boundary-value problem and the stress boundary-value problem have unique solutions, although they are not necessary for uniqueness of solution to either of these problems by itself ${ }^{3}$.

2 Kelvin [1888].

${ }^{3}$ In terms of the Poisson modulus,

the inequalities

$$
\sigma \equiv-\frac{\lambda}{2(\lambda+\mu)},
$$

$$
-1<\sigma<\frac{1}{2}, \quad \mu>0,
$$

are equivalent to (1.1). The inequality $(B)_{1}$ alone implies the validity of either the $\mathrm{C}$-inequalities or their opposites, viz $\mu<0,3 \lambda+2 \mu<0$. The solution of the displacement boundary-value problem is unique, within arbitrary smooth regions, if and only if

$$
\sigma<\frac{1}{2}, \quad \text { or } \quad \sigma>1,
$$

as shown by ERICKSEN $[1957,2]$. (That (C) is sufficient for both existence and uniqueness had been proved by Bogaro [1907]; Mișicu [1953, 2, §16] remarked that these conditions suffice to render the equations of equilibrium strongly elliptic, whence existence and uniqueness of solution follows by application of a known theorem cn differential systems; a far more general result of this kind is given as our Theorem III in $\S 8$, below. Cf. also the discussion by HILL $[1961,4]$.) Uniqueness for the exterior problem, subject to the same inequalities, was established by FICHERA [1950, Cap. I] and by GuRTIN \& Sternberg $[1960,2]$. The range of $\sigma$ consonant with (C) is that in which $\mu$ and $\lambda+2 \mu$ have the same sign; i.e., either (1.2) or their opposites hold.

Bramble \& PAYNe [1962, 2] have shown that a sufficient condition for the stress boundary-value problem, for arbitrary smooth regions inside or outside of a star-shaped surface, to have a solution unique to within an arbitrary rigid motion is

$$
-1<\sigma<1 \text {. }
$$

After some partial results had been established by Bramble \& PAyne [1961, 1], 
They are sufficient but not necessary for the uniqueness of solution to the equations of motion corresponding to assigned initial displacements and velocities upon the bounding surface ${ }^{4}$.

E. Wave speeds. The C-inequalities are sufficient but not necessary in order that the squared speeds of all possible weak waves ${ }^{5}$ be positive. Necessary and sufficient conditions, again ${ }^{6}$, are the less restrictive inequalities (1.2), provided $\varrho>0$.

In the finite theory, arguments of all these types have been tried. HADAMARD 7 gave a definition of stability, from which he derived as a consequence inequalities that are necessary and sufficient for the squared speeds of all kinds of waves to be real. DuHEM ${ }^{8}$ proposed a somewhat different definition of stability, which he showed to imply the same conclusion in regard to waves. Truesdell ${ }^{9}$ used an energy argument to derive an inequality for incompressible isotropic materials; ERICKSEN ${ }^{10}$ found an alternative interpretation for TRUESDELL's inequality in terms of waves; BAKER \& ERICKSEN ${ }^{11}$ were the first to introduce a fairly general statical condition, to which we shall refer more specifically in §3; ERICKSEN \& TOUPIN ${ }^{12}$ found some connections between uniqueness, stability in HADAMARD's sense, and the propagation of waves; BARTA ${ }^{13}$ proposed and explored some static inequalities; a minimum principle for the strain-energy function was proposed

[1962,3], Mindin [unpublished] has proved that the inequalities (D) are also necessary for uniqueness of solution to the stress boundary-value problem, within a general region.

The range of common validity of $(D)$ and $(C)$ is given by $(B)_{1}$. Thus follows the result stated in the text above.

$A$ fortiori, the classical inequality $(\mathrm{B})_{1}$ cannot be weakened if the general mixed boundary-value problem, for arbitrary smooth domains, is to have a solution unique to within a rigid motion (or, if the displacement is prescribed at as many as three non-collinear points, unique).

A general existence theory, subject to the inequalities (C) for the displacement boundary-value problem and to the inequality $(B)_{1}$ for the others, is constructed, for the exterior as well as for the interior problem, in a remarkable but apparently little-known memoir of FICHERA [1950].

4 Gurtin \& Sternberg $[1961,2]$ show that for uniqueness it suffices that the two squared wave-speeds be positive. That is, $\mu / \varrho>0$ and $(\lambda+2 \mu) / \varrho>0$. If $\varrho>0$, this condition reduces to (1.2); if $\varrho \neq 0$, it is equivalent to $(\mathrm{C})$ in the preceding footnote.

5 I.e., singular surfaces of second or higher order, or plane infinitesimal oscillations.

6 The connection between wave propagation and stability as defined under $\mathrm{C}$ was seen by KELVIN [1888]: "Surely, then, if there is a real finite propagational velocity for each of the two kinds of wave-motion, the equilibrium must be stable!" The matter is not really so simple as this. In the finite theory, there are various plausible but different definitions of stability, and there are many kinds of waves.

? [1903, भT 269-271].

$8[1905]$.

- [1952, §41]. We state his result below in $\S 3$, footnote 4 .

While nearly every Italian paper on finite elasticity mentions inequalities, those proposed seem to refer only to the natural state or to special hyperelastic materials. For a specimen, see Signorivi [1949, Cap. I, §1, ๆ 4 and Cap. II, §1, ๆף 5, 7].

10 [1953].

$11[1954,1]$.

$12[1956,1]$.

$13[1957,1]$. In the last footnote to $\S 4$ we state his proposal and give reasons for considering it unsatisfactory. 
by Coleman $\&$ NOLI ${ }^{14}$ and was shown by them to imply not only the classical equations but also a definite criterion of convexity for the stored-energy function and to lead naturally to a definition of stability different from HADAMARD's; Coleman $^{15}$ later showed this minimum principle to be equivalent to a work theorem; and TRUESDELL ${ }^{16}$, after constructing a general theory of wave propagation, was able to interpret BAKER \& ERICKSEN's conditions as well as some of Coleman \& Noll's results directly in terms of wave speeds.

It is our purpose here to follow more deeply the first and simplest kind of argument, beginning from qualitative expectations of static response. Our first goal is to find a static motivation for the inequalities of COLEMAN \& Noll. While most previous work has concerned only hyperelastic materials, defined as those for which the stress-strain relation is derivable from a stored-energy function ${ }^{17}$, here we extend the argument so far as possible to include general elastic materials, defined as those for which the stress is a function of the strain and rotation from a fixed reference configuration. We presume the reader to be already familiar with the elements of these two theories ${ }^{18}$. Our second goal is to formulate for elasticity inequalities that ensure physically natural response, to an extent corresponding to the consequences of the COLEMAN-NoLL condition in hyperelasticity.

Some of the general conclusions of our study are collected and repeated at the end of the paper. Readers not already expert in the general theory of elasticity may be well advised to turn directly to that summary before entering the necessarily somewhat intricate formal work.

\section{List of formulae from the theory of elasticity}

An elastic material is defined by the constitutive equation

$$
T^{k \alpha}=x_{, \beta}^{k} \mathfrak{t}^{\alpha \beta}(\boldsymbol{C}),
$$

where $T^{k \alpha}$ is the first Piola-Kirchhoff stress tensor, $x^{k}{ }_{\alpha \alpha}$ is the deformation gradient, $t^{\beta \alpha}=\mathrm{t}^{\alpha \beta}$ and is formally symmetrized in the components $C_{\alpha \beta}=g_{k m} x^{k},{ }_{\alpha} x^{m}{ }_{, \beta}$ of the symmetric deformation tensor $\boldsymbol{C}$, so that

$$
\frac{\partial t^{\alpha \beta}}{\partial C_{\gamma \delta}}=\frac{\partial t^{\alpha \beta}}{\partial C_{\delta \gamma}} .
$$

Only general elastic materials, not subject to any internal constraint such as incompressibility, are considered in this paper.

$14[1959, \S 8]$. It should be remarked that their principles cover also changes of entropy and temperature, which are not considered in the present study. The special case of their principle for isothermal or isentropic deformations is stated below as our equation (5.1).

$15[1962,4]$.

$16[1961,6, \S 10]$. This work was influenced by an unpublished lecture of ToupIN (1961).

17 However, Baker \& ERICKSEN $[1954,1]$ made no essential use of a storedenergy function, and TRUESDELL's analysis [1961,6] of wave motions expressly avoids it.

${ }_{18}$ A simple account, sufficient for the purposes of the present paper, is given in $[1960,3, \S 303]$. 
An elastic material is hyperelastic if and only if the functions $\mathfrak{t}^{\alpha \beta}()$ satisfy the integrability conditions

$$
\frac{\partial \mathbf{t}^{\alpha \beta}}{\partial C_{\gamma \delta}}=\frac{\partial t^{\gamma \delta}}{\partial C_{\alpha \beta}},
$$

in which case there exists a stored-energy function $\Sigma(\boldsymbol{C})$ such that

$$
\mathrm{t}^{\alpha \beta}=2 \frac{\partial \Sigma(\boldsymbol{C})}{\partial C_{\alpha \beta}} .
$$

We avoid using the relations (2.3) and (2.4) whenever possible, and when we do use them, we remark explicitly that the results so obtained refer to hyperelastic rather than to more general elastic materials.

The Cauchy stress tensor $\boldsymbol{t}$ is related as follows to $\boldsymbol{T}$ :

where

$$
t^{k m}=j^{-1} x^{m},{ }_{\alpha} T^{k \alpha},
$$

$$
j \equiv \frac{\varrho}{\varrho_{0}}=\sqrt{\operatorname{det}\left\|C_{\beta}^{\alpha}\right\|} .
$$

For an isotropic elastic material, $\boldsymbol{t}$ is an isotropic function of $\boldsymbol{C}$, so that

$$
t_{m}^{k}=\jmath_{0} \delta_{m}^{k}+\jmath_{1} B_{m}^{k}+\lambda_{2} B_{p}^{k} B_{m}^{\phi},
$$

where $\boldsymbol{B}$ is the reciprocal deformation tensor, given as follows:

$$
B^{k m}=g^{\alpha \beta} x^{k},{ }_{\alpha} x^{m}, \beta,
$$

and where the scalar coefficients $\lambda_{\Gamma}$ are functions of the principal invariants I, II, and III of $\boldsymbol{B}$. Equivalently,

$$
t_{m}^{k}=\beth_{0} \delta_{m}^{k}+\beth_{1} B_{m}^{k}+\beth_{-1}{ }_{B_{m}^{k}}^{-1}
$$

In a principal co-ordinate system, (2.6) and (2.7) assume the forms

$$
\begin{aligned}
t_{a} & \left.=\lrcorner_{0}+\nu_{1} v_{a}^{2}+\right\lrcorner_{2} v_{a}^{4}, \\
& =\beth_{0}+\beth_{1} v_{a}^{2}+\beth_{-1} v_{a}^{-2},
\end{aligned}
$$

where $v_{a}$ is the principal stretch in the direction along which $t_{a}$ is the principal stress.

For any elastic material, set

$$
A_{r s}^{\alpha \beta} \equiv \frac{\partial T_{r}^{\alpha}}{\partial x_{, \beta}^{s}}, \quad B^{k m p q} \equiv \frac{\varrho}{\varrho_{0}} x^{m}{ }_{, \alpha} x_{, \beta}^{q,} g^{k r} g^{p s} A_{r s}^{\alpha \beta},
$$

and for an isotropic elastic material set ${ }^{1}$

$$
\begin{aligned}
& T_{p q}^{k m} \equiv \frac{\partial t^{k m}}{\partial B^{p q}}=T_{p q}^{m k}=T_{q p}^{k m} \\
& =\frac{1}{2} \lambda_{1}\left(\delta_{p}^{k} \delta_{q}^{m}+\delta_{p}^{m} \delta_{\xi}^{k}\right)+\frac{1}{2} \eta_{2}\left(\delta_{p}^{k} B_{q}^{m}+\delta_{p}^{m} B_{q}^{k}+\delta_{q}^{k} B_{p}^{m}+\delta_{q}^{m} B_{p}^{k}\right)+ \\
& +g^{k m}\left[\frac{\partial \boldsymbol{\lambda}_{0}}{\partial \mathrm{I}} g_{p q}+\frac{\partial \boldsymbol{\lambda}_{0}}{\partial \mathrm{II}}\left(\mathrm{I} g_{p q}-B_{p q}\right)+\mathrm{III} \frac{\partial \boldsymbol{\lambda}_{1}}{\partial \mathrm{III}} B_{p q}\right]+ \\
& +B^{k m}\left[\frac{\partial \boldsymbol{I}_{1}}{\partial \mathrm{I}} g_{p q}+\frac{\partial \boldsymbol{I}_{1}}{\partial \mathrm{II}}\left(\mathrm{I} g_{p q}-B_{p q}\right)+\mathrm{III} \frac{\partial \mathcal{I}_{1}}{\partial \mathrm{III}} B_{p q}\right]+ \\
& +B_{r}^{k} B^{r m}\left[\frac{\partial \boldsymbol{M}_{2}}{\partial \mathrm{I}} g_{p q}+\frac{\partial \boldsymbol{\lambda}_{2}}{\partial \mathrm{II}}\left(\mathrm{I} g_{p q}-B_{p q}\right)+\operatorname{III} \frac{\partial \boldsymbol{\lambda}_{2}}{\partial \mathrm{III}} B_{p q}\right] \text {. }
\end{aligned}
$$

${ }^{1}$ The expressions $(2.10)_{4}$ and (2.11) were given by TRUeSDELL [1961, 6, Eqq. (7.5) and $(12.5 \mathrm{E})]$ 
In a principal co-ordinate system

$$
T^{11}{ }_{11}=\frac{\partial t_{1}}{\partial v_{1}^{2}}, \quad T^{11}{ }_{22}=\frac{\partial t_{1}}{\partial v_{2}^{2}}, \ldots \quad T_{12}^{12}=\frac{t_{1}-t_{2}}{2\left(v_{1}^{2}-v_{2}^{2}\right)}, \ldots
$$

while all components $T_{p q}^{k m}$ not obtainable from one of these three by permutation of indices are zero. In any co-ordinate system

$$
B_{p}^{k m}{ }_{p}^{q}=t^{k m} \delta_{p}^{q}-t^{k q} \delta_{p}^{m}+2 T_{p s}^{k m} B^{q s} .
$$

In an isotropic material subject to homogeneous deformation from its natural state, consider a block with faces perpendicular to the principal axes of strain and stress. The forces acting upon these faces are tensions of magnitude $t_{a}$ per unit area. If the block before deformation was a unit cube, the total forces acting upon its faces in the deformed state are given by the formulae

or

$$
T_{1}=v_{2} v_{3} t_{1}, \quad T_{2}=v_{3} v_{1} t_{2}, \quad T_{3}=v_{1} v_{2} t_{3},
$$

$$
T_{a}=\frac{\varrho}{\varrho_{0}} \cdot \frac{t_{a}}{v_{\mathfrak{a}}} .
$$

In a pure strain, it is possible to select a common Cartesian co-ordinate system such that the matrix of deformation gradients $x^{m}{ }_{, \beta}$ is a diagonal matrix; in such a system, the matrix of the Piola-Kirchhoff stress tensor $T_{k}^{\alpha}$ is also diagonal, and its entries are the quantities $T_{\alpha}$. In a general deformation of an isotropic elastic material it is possible to choose co-ordinates such that $\left\|T_{k}^{\alpha}\right\|=\left[\operatorname{diag}\left(T_{k}\right.\right.$, $\left.\left.T_{2}, T_{3}\right)\right] \boldsymbol{R}$, where $\boldsymbol{R}$ is a rotation matrix ${ }^{2}$. Accordingly, we shall call the $T_{a}$ the principal forces.

In isotropic materials the principal stresses, hence also the principal forces, are functions of the principal stretches:

$$
\begin{aligned}
t_{a} & =t_{a}\left(v_{1}, v_{2}, v_{3}\right), \\
T_{a} & =T_{a}\left(v_{1}, v_{2}, v_{3}\right) .
\end{aligned}
$$

More specifically, isotropy of the elastic material implies that a single function $f\left(v_{1}, v_{2}, v_{3}\right)$ serves to define all three functions $T_{a}()$ in $(2.15)$ :

where

$$
T_{1}=f\left(v_{1}, v_{2}, v_{3}\right), \quad T_{2}=f\left(v_{2}, v_{3}, v_{1}\right), \quad T_{3}=f\left(v_{3}, v_{1}, v_{2}\right),
$$

$$
f(x, y, z)=f(x, z, y) \text {. }
$$

For an isotropic hyperelastic material the stored energy may be taken as a symmetric function of the principal stretches: $\tilde{\Sigma}\left(v_{1}, v_{2}, v_{3}\right)=\Sigma(\boldsymbol{C})$, and (2.4) is equivalent to the statement that

$$
f\left(v_{1}, v_{2}, v_{3}\right)=\frac{\partial \widetilde{\Sigma}\left(v_{1}, v_{2}, v_{3}\right)}{\partial v_{1}}
$$

also, the Jacobian matrix of $(2.15)_{2}$, having the components

$$
J_{a b} \equiv \frac{\partial T_{a}}{\partial v_{b}},
$$

2 The proof follows easily by use of $(2.5)$ and of the dual of $[1960,3,(37.9)]$. 
is a symmetric matrix for all values of the stretches. From (2.16) and (2.17) it follows that even for elastic materials that are not hyperelastic, $\left\|J_{a b}\right\|$ is symmetric whenever $v_{1}=v_{2}=v_{3}$. More generally, $J_{a b}=J_{b a}$ whenever $v_{a}=v_{b}$. (This particular symmetry, which follows from isotropy alone, accounts for the well known but curious fact that in the linearized theory of elasticity, every isotropic material is hyperelastic, or in other words, that isotropy in the linearized theory implies the existence of a stored-energy function.) If we adopt the usual assumption that the functions $T_{a}()$ are continuously differentiable, we see that an elastic material is hyperelastic if and only if

$$
J_{a b}=J_{b a}
$$

for all values of the stretches $v_{c}$. (Even in a hyperelastic material, $\partial t_{a} / \partial v_{b} \neq \partial t_{b} / \partial v_{a}$ in general, but the condition $v_{a}=v_{l}$ is sufficient that $\partial t_{a} / \partial v_{b}=\partial t_{b} / \partial v_{a}$ even when there is no stored-energy function.)

A configuration in which the stress vanishes is called a natural state of the elastic material. If such a state exists, we may choose it as the reference configuration, and, having done so, we conclude from (2.1) and (2.15) that

$$
\mathbf{t}(\mathbf{1})=0, \quad t_{a}(1,1,1)=T_{a}(1,1,1)=0 .
$$

Some elastic materials do not have a natural state. For example, an elastic fluid, defined by the condition

$$
t_{a}=-p\left(v_{1} v_{2} v_{3}\right), \quad a=1,2,3,
$$

is an isotropic hyperelastic material in which the stress never vanishes, provided the function $p()$ be subject to the condition $p(\xi)>0$ if $0<\xi<\infty$. The general considerations in this study do not presume the existence of a natural state, and special notice will be called to such particular results as do require one. Among such special results are the $\mathrm{C}$-inequalities.

\section{Some plausible static inequalities for isotropic materials}

In the theory of finite elastic strain it is clearly of no use to consider special deformations such as simple tension, simple shear, and uniform dilation, since special relations hold among the stretches occurring in those deformations, so no restriction upon general functions of those stretches can possibly result by laying down conditions for these cases. The approach numbered A1 in the discussion just following (1.1) is effective in the classical linearized theory only because the response of the material is specified in terms of constants, viz $\lambda$ and $\mu$, instead of functions of the stretches. Thus approach A 1 would be fruitless for us.

Not so for A2. We lay down some simple conditions that we may expect intuitively to be just for any isotropic material. At the beginning we limit our study to isotropic materials because when more general material symmetries are present the intuitive picture is no longer clear.

$\alpha$. The $P-C$ inequality. The condition that the volume of a compressible isotropic material should be decreased by pressure but increased by tension is expressed by requiring the hydrostatic tension $t$ to be a strictly increasing 
function of the stretch, $v$ :

$$
(\bar{t}-t)(\bar{v}-v)>0, \quad \bar{v} \neq v,
$$

where $t=t(\bar{v})$. In particular, if the material has a natural state, so that $t(1)=0$, (3.1) implies that $t(v-1)>0$ if $v \neq 1$. For a general deformation, there are many inequalities that are invariant and that reduce to this latter one in the special case of hydrostatic stress. An example is the set of three inequalities $t_{a}\left(v_{a}-1\right)>0, a=1,2,3$, stating that each principal stress is a pressure or a tension according as the corresponding principal stretch is a contraction or an elongation, but these inequalities are too strong. It should be possible in general, as it is in the linearized theory of elasticity, that a severe pull in one direction may result in transverse contraction even when some transverse tension is applied. It is reasonable, however, to demand an inequality of this type in mean, and we are led to consider either or both of the following:

$$
\sum_{a=1}^{3} t_{a}\left(v_{a}-1\right)>0, \quad \sum_{a=1}^{3} T_{a}\left(v_{a}-1\right)>0,
$$

if not all $v_{a}=1$. We shall see later that $(3.2)_{2}$ is implied by certain more general and plausible conditions that do not always lead to (3.2) $)_{1}$. In terms of principal stresses, (3.2) ${ }_{2}$ can be written

$$
\sum_{a=1}^{8} t_{a} \frac{v_{a}-1}{v_{a}}>0 \text { if not all } v_{a}=1 .
$$

Thus our intuition does not suggest, in this instance, which of the following two strain measures to select as a basis for inequalities:

$$
\begin{aligned}
& v_{a}-1=\frac{\text { change in length }}{\text { initial length }}, \\
& \frac{v_{a}-1}{v_{a}}=\frac{\text { change in length }}{\text { final length }} .
\end{aligned}
$$

Using the letters P-C to recall "pressure-compression", we shall refer to (3.2) as the $P$ - $C$ inequality. An invariant form of it, valid in any co-ordinate system, is

Another form is

$$
\operatorname{tr}\left[\boldsymbol{t}\left(1-B^{-1}\right)\right]>0 \text {. }
$$

$$
\sum_{a=1}^{3} t_{a}>\frac{\varrho_{0}}{\varrho} \sum_{a=1}^{3} T_{a} \text {. }
$$

Note that only for materials having a natural state is the P-C inequality to be expected. E.g. for an elastic fluid, while (3.1) ought to be satisfied, the P-C inequality $(3.2)_{2}$ certainly is not. Neither is the inequality (3.2), in general.

$\beta$. The T-E inequalities. It is natural to expect that when a cube of isotropic material is lengthened along one principal direction while its faces parallel to that direction are kept fixed, the tensile force must be increased, but to shorten it, the tensile force must be reduced. This condition may be expressed in either of the following equivalent forms:

$$
\left.\left(\bar{T}_{a}-T_{a}\right)\left(\bar{v}_{a}-v_{a}\right)>0, \quad \bar{t}_{a}-t_{a}\right)\left(\bar{v}_{a}-v_{a}\right)>0,
$$

provided $\bar{v}_{b}=v_{b}$ if $b \neq a$. Here we are using the notations $\bar{T}_{a}=T_{a}\left(\bar{v}_{1}, \bar{v}_{2}, \bar{v}_{3}\right)$, etc. From these conditions it follows, when $T_{a}()$ is assumed continuously differenti- 
able, that

$$
\frac{\partial T_{a}}{\partial v_{a}}>0, \quad \frac{\partial t_{a}}{\partial v_{a}}>0,
$$

except upon a nowhere dense set. Conversely, if we require (3.8) to hold everywhere, then (3.7) follows. Using the letters T-E to recall "tension-extension", we shall refer to (3.7) as the T-E inequalities, while the stronger conditions (3.8) will be called the $T-E^{+}$inequalities ${ }^{1}$.

$\gamma$. The IFS condition. It may be reasonable to expect that if given pairs of opposing normal forces of magnitudes $T_{a}$ are applied to the faces of a unit cube of isotropic elastic material, one and only one pure homogeneous deformation will result. This condition asserts that the equations $T_{a}=T_{a}\left(v_{1}, v_{2}, v_{3}\right)$ be uniquely invertible. Using "IFS" to recall "invertibility of force-stretch", we may name this requirement the IFS condition. Under the usual assumption that the stressstrain relations are continuously differentiable, $J_{a b}()$ is a continuous function, and the IFS condition implies that

$$
\operatorname{det}\left\|J_{a b}\right\| \neq 0
$$

except upon a nowhere dense set ${ }^{2}$. Conversely, if (3.9) holds everywhere, the IFS condition holds in virtue of a classical theorem of analysis. We shall refer to (3.9) as the IFS $S^{+}$condition.

The stress-stretch relations (2.45) are not necessarily invertible when (3.9) holds, nor is it generally reasonable to expect them to be so. For example, when the elastic material is a fluid, so that $t_{a}=-p\left(v_{1} v_{2} v_{3}\right)$, it is obviously impossible to solve for the $v_{b}$ as functions of the principal stresses, yet the relations for principal forces may be invertible. To choose a case, consider the perfect gas law, $p\left(v_{1} v_{2} v_{3}\right)=K /\left(v_{1} v_{2} v_{3}\right)$, which leads to the unique inverse relations $v_{a}=K / T_{a}, a=1,2,3$. Here again we see evidence in favor of requiring regular or simple behavior of the principal forces rather than of the principal stresses.

$\delta$. The E-T inequalities. Granted that we may solve for the principal stretches as functions of the principal forces $T_{a}$, it is reasonable to expect that if one pair of opposing normal forces on a homogeneously strained block with faces perpendicular to the principal axes are increased in magnitude, while the remaining pairs are kept fixed, the block will lengthen in the corresponding direction. This condition may be expressed in the form

$$
\left(\bar{T}_{a}-T_{a}\right)\left(\bar{v}_{a}-v_{a}\right)>0
$$

provided $\bar{T}_{b}=T_{b}$ if $b \neq a$. This is a condition on the three functions $v_{a}\left(T_{a}, T_{b}, T_{c}\right)$ when $T_{b}$ and $T_{c}$ are held constant, while the formally similar condition $(3.7)_{1}$ restricts the three functions $T_{a}\left(v_{a}, v_{b}, v_{c}\right)$ when $v_{b}$ and $v_{c}$ are held constant. Thus, clearly, neither condition implies the other in general. From (3.10) we infer, if $v_{a}()$ is assumed continuously differentiable, that

$$
\frac{\partial v_{a}}{\partial T_{a}}>0
$$

${ }^{1}$ It has been shown by TRUeSDell $[1961,6, \S \S 7,10]$ that the T-E ${ }^{+}$inequalities are necessary and sufficient conditions that the squared speeds of all principal longitudinal waves be positive. If only the $\mathrm{T}-\mathrm{E}$ inequalities are required, material longitudinal disturbances become possible, i.e., non-propagating surfaces across which the density gradient is discontinuous.

2 Bernstein \& Toupin [1962, 1, Theorem VI]. 
except on a nowhere dense set. Conversely, if we require (3.11) to hold everywhere, (3.10) follows. Using the letters E-T to recall "extension-tension", we refer to (3.10) as the E-T inequalities, while the stronger conditions (3.11) will be called the $E-T^{+}$inequalities.

$\varepsilon$. The $O-F$ inequalities. Consider again a block of isotropic material supposed to be in equilibrium subject to pairs of equal and oppositely directed normal forces acting upon its faces. It may be reasonable to expect that the greater stretch will occur in the direction of the greater force. Thus we are led to require that

$$
\left(T_{a}-T_{b}\right)\left(v_{a}-v_{b}\right)>0 \text { if } v_{a} \neq v_{b} .
$$

For hyperelastic materials this inequality was first proposed by ColEMAN \& NoLL ${ }^{3}$, who derived it as a consequence of the general inequality we shall discuss below in $\$ 5$. Using the letters $\mathrm{O}-\mathrm{F}$ to recall "ordered forces", we shall refer to (3.12) as thc $O-F$ inequalities. From (2.16) and (2.17) we see that if $v_{a}=v_{b}$, it necessarily follows that $T_{a}=T_{b}$. Thus no particular assumption is needed in order to generalize the $\mathrm{O}-\mathrm{F}$ inequalities so as to hold if the principal stretches coalesce. The assumption that $\left(T_{a}-T_{b}\right)\left(v_{a}-v_{b}\right) \geqq 0$, where equality holds if and only if $v_{a}=v_{b}$, is equivalent to (3.12).

$\zeta$. The B-E inequalities. Inequalities similar to (3.12), but referring to principal stresses rather than to principal forces, were first suggested by BAKER \& ERICKSEN 4 . Specifically, they proposed that the greater principal stress occur always in the direction of the greater principal stretch:

$$
\left(t_{a}-t_{b}\right)\left(v_{a}-v_{b}\right)>0 \text { if } v_{a} \neq v_{b} .
$$

We shall refer to these as the $B$-E inequalities ${ }^{5}$. Equivalently, by (2.16), (2.17), and (2.13), we may write (3.13) in the form $\left(t_{a}-t_{b}\right)\left(v_{a}-v_{b}\right) \geqq 0$, where equality holds if and only if $v_{a}=v_{b}$.

Each of the foregoing inequalities may be expressed as a restriction on the response coefficients $\lambda_{\Gamma}$ or $a_{\Gamma}$ occurring in (2.6)-(2.8). Since

$$
t_{a}-t_{b}=\left(v_{a}^{2}-v_{b}^{2}\right)\left(\beth_{1}-\frac{1}{v_{a}^{2} v_{b}^{2}} \beth_{-1}\right)
$$

3 [1959, §12].

$4[1954,1]$.

5 BAKer \& ERICKSEN [1954, 1] showed that, for incompressible hyperelastic materials, (3.13) implies that the stored-energy function satisfies the inequalities

$$
\frac{\partial \Sigma}{\partial \mathrm{I}}+v_{a}^{2} \frac{\partial \Sigma}{\partial \mathrm{II}}>0, \quad a=1,2,3 \text {. }
$$

These conditions had been proposed by TRUESDELL [1952, $\S 41]$, who showed them to be necessary and sufficient that positive work be required in order to increase one principal extension while holding another one fixed. It was then shown by ERICKSEN [1953, 1] that TRUESDELL'S inequalities are in turn necessary and sufficient for the squared speeds of all weak principal waves (necessarily transverse) in an incompressible isotropic hyperelastic material to be positive. It has been shown recently by TRUESDELL $[1961,6, \S \S 7,10]$ that, in a compressible isotropic elastic material, whether or not it be hyperelastic, the B-E inequalities are necessary and sufficient conditions that the squared speeds of all transverse principal waves-be positive. 
the B-E inequalities are equivalent to ${ }^{5}$

$$
y_{1}-\frac{1}{v_{a}^{2} v_{b}^{2}} y_{-1}>0 \text { if the } v_{a} \text { are distinct, }
$$

while the $\mathrm{O}-\mathrm{F}$ inequalities are equivalent to

$$
-v_{a} v_{b} z_{0}+\beth_{1}-v_{a}^{2}+v_{a} \frac{v_{b}+v_{b}^{2}}{v_{a}^{3} v_{b}^{3}} \cdot \beth_{-1}>0
$$

if the $v_{a}$ are distinct. Accordingly, a simple set of inequalities implying both the $\mathrm{B}-\mathrm{E}$ inequalities and the $\mathrm{O}-\mathrm{F}$ inequalities is

$$
\eta_{1} \leq 0, \quad \eta_{1}>0, \quad \beth_{-1} \leqq 0 .
$$

To the limited extent that experimental data are available, they seem to support these inequalities, which shall therefore be called the E-inequalities, where $\mathrm{E}$ is a mnemonic for "empirical". No theoretical motivation has been found for them, however, beyond their logical relation to the B-E and O-F inequalities, which may be abbreviated as follows:

$$
\mathrm{E} \Rightarrow \mathrm{B}-\mathrm{E} \& \mathrm{O}-\mathrm{F} \text {. }
$$

In the next section we shall establish some less obvious connections between the various inequalities stated.

\section{Some logical connections among the static inequalities}

In the linearized theory the several inequalities developed in $\$ 3$ reduce to the following forms ${ }^{1}$ :

$$
\begin{array}{ll}
\text { P-C: } & \mu>0, \quad 3 \lambda+2 \mu>0 \\
\text { T-E, T-E }, \text { E-T, E-T }: & \lambda+2 \mu>0 \\
\text { IFS, IFS }: & \mu(3 \lambda+2, \mu) \neq 0 \\
\text { O-F, B-E: } & \mu>0 .
\end{array}
$$

6 Baker \& Eiricksien $[1954,1]$.

1 In the linearized theory the E-inequalities have no precise expression, but they are sufficient that $\mu>0$. Note that the T-E and E-T inequalities in the linearized theory are not the same as the requirement that the modulus of extension be positive:

$$
\frac{\mu(3 \lambda+2 \mu)}{\lambda+\mu}>0 .
$$

In simple extension along the direction of $v_{1}$, the transverse stretches $\eta_{2}$ and $\dot{v}_{3}$ are adjusted so that $t_{2}=t_{3}==0$. Therefore

$$
v_{2}-1=v_{3}-1=-\underset{\lambda}{2(\lambda+\mu)}\left(v_{1}-1\right)=-\sigma\left(v_{1}-1\right),
$$

so that $v_{2}$ and $v_{3}$ cannot be held constant when $v_{1}$ is varied. In the finite theory we cannot even formulate definitely an analogue of $(G)$ because a formula such as $(\mathrm{H})$, giving the transverse-contraction ratio $\sigma$ explicitly in terms of material constants or functions, cannot be found. $C /$. the discussion of simple extension by Truesdel [1952, §42D]. 
From these forms we may read off several non-implications:

$$
\begin{aligned}
& \mathrm{O}-\mathrm{F} \Rightarrow \mathrm{\Rightarrow}-\mathrm{C} \text { or } \mathrm{T}-\mathrm{E} \text { or } \mathrm{E}-\mathrm{T}, \\
& \mathrm{B}-\mathrm{E}=\Rightarrow \mathrm{P}-\mathrm{C} \text { or } \mathrm{T}-\mathrm{E} \text { or } \mathrm{E}-\mathrm{T} .
\end{aligned}
$$

The example $\lambda=-\mu, \mu>0$ shows that

$$
\text { (T-E or E-T) \& (O-F or B-E) } \Rightarrow \Rightarrow \text { P-C. }
$$

Either from the table (4.1) or directly from (3.1) we see that in the linearized theory the P-C inequality is equivalent to the requirement that the work done in effecting any non-rigid deformation be positive. Hence the P-C inequality implies all the requirements customarily imposed in the linearized theory ${ }^{2}$, as may be verified also from the table (4.1): $\mathrm{P}-\mathrm{C} \Rightarrow \mathrm{C}$. In a general deformation, however, the P-C inequality does not have any evident energetic meaning, nor does it imply any of the others considered here. Neither do the E-inequalities imply the P-C inequalities.

The inequalities listed in $\$ 3$ fall into three types: those that relate a single stress or force to the corresponding stretch, those that relate pairs of stresses to pairs of stretches, and those that relate all three of each. We shall discuss now the three types separately, beginning with the second, consisting only in the $\mathrm{O}-\mathrm{F}$ and $\mathrm{B}-\mathrm{E}$ inequalities.

While the $\mathrm{O}-\mathrm{F}$ and $\mathrm{B}-\mathrm{E}$ inequalities are equivalent in the linearized theory, in general

$$
\mathrm{O}-\mathrm{F} \Rightarrow \mathrm{B}-\mathrm{E} \text { and } \mathrm{B}-\mathrm{E} \Rightarrow \mathrm{O}-\mathrm{F} \text {. }
$$

For example, if $T<0$ the triples $(T, 2 T, 3 T)$ and $\left(v, \frac{1}{2} v, \frac{1}{4} v\right)$ satisfy the O-F inequalities, but the principal stresses are $\left(8 T / v^{2}, 8 T / v^{2}, 6 T / v^{2}\right)$, which do not satisfy the B-E inequalities. There is, however, an intimate connection between the two inequalities. To see it, we consider the function

$$
f(r)=r \frac{r^{2}+3}{1+3 r^{2}}
$$

and observe that since $f^{\prime}(r)=3\left(r^{2}-1\right)^{2} /\left(1+3 r^{2}\right)^{2}, f$ is monotone increasing; hence, since also $f(0)=0$ and $f(1)=1$, it follows that $0<f(r)<1$ when $0<r<1$, while $f(r)>1$ when $r>1$. Let the principal stretches be distinct and ordered by number, so that $v_{1}>v_{2}>v_{3}$. Assume that the O-F inequalities hold. Set $r=v_{1} / v_{2}$, and multiply the inequality $f(r)>1$ by the inequality $T_{1}>T_{2}$. If we assume further that $T_{1} \geqq 0$, it follows that

Hence

$$
\frac{v_{1}\left(v_{1}^{2}+3 v_{2}^{2}\right)}{v_{2}\left(v_{2}^{2}+3 v_{1}^{2}\right)} T_{1}>T_{2} .
$$

Equivalently, by (2.13),

$$
\frac{v_{1}\left(v_{1}^{2}+3 v_{2}^{2}\right) T_{1}-v_{2}\left(v_{2}^{2}+3 v_{1}^{2}\right) T_{2}}{v_{1}^{2}-v_{2}^{2}}>0 .
$$

$$
\frac{t_{1}-t_{2}}{v_{1}-v_{2}}>\frac{1}{2}\left(t_{1}+t_{2}\right) \frac{v_{1}+v_{2}}{v_{1}^{2}+v_{2}^{2}}
$$

2By "the linearized theory" we mean that of CAUCHX, in which there may be as many as 36 distinct elasticities. Of course the P-C inequality does not imply that there is a stored-energy function. 
subject to our assumptions, namely, that the O-F inequalities hold and that $t_{1} \geqq 0$. Similarly, if $t_{2} \geqq 0$, the $\mathrm{O}-\mathrm{F}$ inequalities imply that

$$
\frac{t_{2}-t_{3}}{v_{2}-v_{3}}>\frac{1}{2}\left(t_{2}+t_{3}\right) \frac{v_{2}+v_{3}}{v_{2}^{2}+v_{8}^{2}}
$$

The right-hand sides of (4.8) and (4.9) are non-negative if $t_{1}+t_{2} \geqq 0$ and $t_{2}+t_{3} \geqq 0$. In order to derive (4.8) and (4.9) we have assumed that $t_{1} \geqq 0$ and $t_{2} \geqq 0$, although $t_{3}$ may be negative. Thus we may summarize the foregoing analysis as follows: In $a$ state of stress such that $v_{1}>v_{2}>v_{3}$ and $t_{1} \geqq 0, t_{2} \geqq 0, t_{2}+t_{3} \geqq 0$,

$$
\mathrm{O}-\mathrm{F} \Rightarrow \mathrm{B}-\mathrm{E} \text {. }
$$

States of pure tension are included as a special case ${ }^{3}$. The cases when two or three principal stretches coalesce need no special mention, since the corresponding principal stresses and principal forces coalesce also, irrespective of any inequalities.

Again let the principal stretches be ordered by number, so that $v_{1}>v_{2}>v_{3}$, but assume that the B-E inequalities hold; thus $-t_{3}>-t_{2}>-t_{1}$. Assume that $t_{3} \leqq 0$. Setting $r=v_{2} / v_{3}$ and multiplying the inequality $f(r)>1$ by the inequality $-t_{3}>-t_{2}$, we infer that

Hence

$$
v_{2}\left(v_{2}^{2}+3 v_{3}^{2}\right) t_{3}<v_{3}\left(v_{3}^{2}+3 v_{2}^{2}\right) t_{2}
$$

$$
\frac{T_{2}-T_{3}}{v_{2}-v_{3}}>-\frac{1}{2}\left(T_{2}+T_{3}\right) \frac{v_{2}+v_{3} v_{3}^{*}}{v_{2}^{2}+v_{3}^{2}}
$$

If $t_{2} \leqq 0$, then $T_{2}+T_{3} \leqq 0$, and (4.12) yields $T_{2}>T_{3}$. In a similar manner we infer that $T_{1}>T_{2}$ if $T_{1}+T_{2} \leqq 0$. Accordingly, we have proved that in a state of stress such that $v_{1}>\dot{v}_{2}>v_{3}$ and $t_{3} \leqq 0, t_{2} \leqq 0, \frac{t_{1}}{v_{1}}+\frac{t_{2}}{v_{2}} \leqq 0$,

$$
\mathrm{B}-\mathrm{E} \Rightarrow \mathrm{O}-\mathrm{F} \text {. }
$$

States of pure pressure are included as a special case.

Further analysis may reveal other connections between the B-E and O-F inequalities, but it is clear that they express, in general, distinct requirements.

Turning now to the inequalities that relate principal forcess or principal stresses to the corresponding stretches, we discuss them in terms of the Jacobian matrix $\left\|J_{a b}\right\|$, where $J_{a b}$ is defined by (2.19). The C-inequalities, as remarked in $\S 1$, are sufficient that $\operatorname{det}\left\|J_{a b}(1,1,1)\right\|>0$. Continuing to adopt the assumption that $J_{a b}()$ is a continuous function of the $v_{c}$, from (3.9) we see that

$$
\mathrm{IFS}^{+} \& \mathrm{C} \Leftrightarrow \operatorname{det}\left\|J_{a b}\right\|>0 \text {. }
$$

Let us now consider conditions under which the matrix $\left\|J_{a b}\right\|$, or, equivalently, the symmetrized matrix $\left\|J_{(a b)}\right\|$, is positive-definite. To begin with, restrict attention to hyperelastic materials, which are characterized by the symmetry conditions $J_{a b}=J_{(a b)}$. If we assume the invertibility of the relations $(2.15)_{2}$,

That. O-F $\Rightarrow$ B-E for states of pure tension was remarked by ColEman \& NolL [1959, §12]. 
the principal minors of $\left\|J_{a b}\right\|$ are

$$
\partial T_{a}, \frac{\partial v_{a}}{\partial v_{a}} \operatorname{det}\left\|J_{b c}\right\|, \operatorname{det}\left\|J_{b c}\right\| .
$$

By $(3.8)_{1},(3.11)$, and (4.14) we infer that

$$
\left.\begin{array}{l}
\mathrm{C} \quad \& \\
\mathrm{IFS}^{+} \& \\
\mathrm{~T}^{-\mathrm{E}^{+}} \& \\
{\mathrm{E}-\mathrm{T}^{+}}^{+}
\end{array}\right\} \Leftrightarrow\left\|_{1} J_{a b}\right\|>0
$$

for all $v_{c}$, where we use the notation " $\|A\|>0$ " to denote the statement that $\|A\|$ is positive-definite. None of the four conditions listed on the left-hand side of the implication is redundant; if we leave off $\mathrm{C}$, there remains the possibility that det $\left\|J_{a b}\right\|<0$, while if we leave off $\mathrm{IFS}^{+}$, it becomes possible that det $\left\|J_{a b}\right\|=0$. etc. Thus we have found a full and minimal set of simple, plausible, purely static conditions equivalent to the statement that $\left\|J_{a b}\right\|>0$, for hyperelastic materials.

This is not the end, however. If $J_{a b}=J_{b a}$, as we are assuming, there exists a stored-energy function $\tilde{\Sigma}\left(v_{1}, v_{2}, v_{3}\right)$ satisfying (2.18), and if $\left\|J_{a b}\right\|>0$, that function is convex for all stretches. Therefore,

$$
\tilde{\Sigma}\left(\bar{v}_{1}, \bar{v}_{2}, \bar{v}_{3}\right)-\tilde{\Sigma}\left(v_{1}, v_{2}, v_{3}\right)-\sum_{a=1}^{3} T_{a}\left(\bar{v}_{a}-v_{a}\right)>0,
$$

provided only that $\bar{v}_{a} \neq v_{a}$ for some $a$. Interchanging the $\bar{v}_{a}$ with the corresponding $v_{a}$ and adding the resulting inequality to (4.17), we infer that

$$
\sum_{a=1}^{3}\left(\bar{T}_{a}-T_{a}\right)\left(\bar{v}_{a}-v_{a}\right)>0,
$$

For a reason that will be made clear in $\$ 5$, we shall call (4.18) the $G C N_{0}$ inequality. Looking back at (3.1), (3.2) $2,(3.7),(3.10)$, and (3.12), we see that the $\mathrm{GCN}_{0}$ inequality is a similar but more general statement. Indeed, it includes them all. If we assume that a natural state exists, by setting $\bar{v}_{a}=1$ we obtain $\bar{T}_{a}=0$ and so reduce $(4.18)$ to $(3.2)_{2}$. If we choose the $\bar{v}_{a}$ as a permutation of the $v_{a}$, because of (2.16) and (2.17) the $\bar{T}_{a}$ are the corresponding permutation of the $T_{n}$, so that (4.18) yields

$$
\sum_{a=1}^{3}\left(T_{\pi(a)}-T_{a}\right)\left(v_{\pi(a)}-v_{a}\right)>0,
$$

provided only that $\pi(a)$ is not the identity permutation and that $v_{a} \neq v_{b}$ if $a \neq b$. Permuting any two of the $v_{c}$ but leaving the other unchanged reduces (4.19) to (3.12). Thus it has been shown that (4.18), here obtained as a consequence of assuming that $\left\|J_{a b}\right\|>0$, implies the P-C and O-F inequalities. Thus, in addition to (4.16) we may now record the following implications, still for hyper-

4 It was shown by Coleman \& Noll $[1959, \S 12]$ that (4.17) implies the O-F inequalities. The argument leading from (4.17) to (4.18), (4.19), and their consequences derived above was.shown to us by NOLL in 1960. 
elastic materials:

$$
\begin{aligned}
& \left\|J_{a b}\right\|>0 \rightarrow\left\{\begin{array}{l}
\mathrm{P}-\mathrm{C} \& \\
\mathrm{IFS}+\& \\
\mathrm{~T}-\mathrm{E}^{+} \& \\
\mathrm{E}-\mathrm{T}^{+} \& \\
\mathrm{O}-\mathrm{F},
\end{array}\right. \\
& \left.\begin{array}{l}
\mathrm{Cr}^{+} \& \\
\mathrm{IFS}^{+} \& \\
\mathrm{~T}_{-} \mathrm{E}^{+} \& \\
\mathrm{E}_{-} \mathrm{T}^{+}
\end{array}\right\} \Rightarrow \mathrm{P}-\mathrm{C} \& \mathrm{O}-\mathrm{F} .
\end{aligned}
$$

(In order to include the P-C inequality in (4.20) and (4.21), one must assume the existence of a natural state, which is not necessary for the other implications.) In other words, if we are willing to accept principal forces rather than principal stresses as the proper variables in terms of which static inequalities are to hold, the following inequalities, all in the strengthened form denoted by a $t^{-}$, suffice:

Classical inequalities (C),

Invertibility of Force-Stretch relations ( $\mathrm{IFS}^{\mathrm{r}}$ ),

Tension-Extension inequalities $\left(\mathrm{T}-\mathrm{E}^{+}\right)$,

Extension-Tension inequalities $\left(\mathrm{E}-\mathrm{T}^{+}\right)$,

since all the other plausible requirements we have suggested follow from them as consequences, by (4.16) and (4.21).

How much of all this can be salvaged for more general elastic materials? First, the $\mathrm{GCN}_{0}$-inequality (4.18) can be stated, and the consequences drawn from it above made no use of a stored-energy function. Moreover, setting $\bar{v}_{2}=v_{2}$, $\bar{v}_{3}=v_{3}$, from (4.18) we derive (3.7). Further, from (4.18) we see that $\bar{T}_{a}=T_{a}$, $a=1,2,3$, is impossible if $v_{a} \neq v_{a}$ for some $a$. That is, the relations $T_{a}=T_{a}\left(v_{1}, v_{2}, v_{3}\right)$ must be uniquely invertible. Consequently we may regard the $v_{a}$ as functions of the $T_{b}$ in (4.18) and thus infer (3.10). In summary, then, we have shown that

$$
\mathrm{GCN}_{0} \Rightarrow\left\{\begin{array}{l}
\text { P-C \& } \\
\text { IFS \& } \\
\text { T-E \& } \\
\text { E-T \& } \\
\text { O-F }
\end{array}\right.
$$

so that the $\mathrm{GCN}_{0}$ inequality implies all the simple statical conditions laid down as plausible in $\$ 3$, irrespective of whether or not there is a stored-energy function. (Again it is necessary to presume the existence of a natural state in order to infer the P-C inequality, but the other implications in (4.22) hold also for materials without a natural state.) We have been unable to show that this implication can be reversed.

Finally we turn to consider properties of the non-symmetric matrix $\left\|J_{a b}\right\|$, which is positive-definite if and only if its symmetric part, $\left\|J_{(a b)}\right\|$, is positive- 
definite. By writing out the principal minors of $\left\|J_{(a b)}\right\|$ we infer that

$$
\left\|J_{a b}\right\|>0 \Leftrightarrow\left\{\begin{array}{l}
\mathrm{T}-\mathrm{E}^{+} \& \\
\left(\frac{\partial T_{a}}{\partial v_{b}}+\frac{\partial T_{b}}{\partial v_{a}}\right)^{2}<4 \frac{\partial T_{a}}{\partial v_{a}} \frac{\partial T_{b}}{\partial v_{b}} \& \\
\operatorname{det}\left\|J_{(a b)}\right\|>0 .
\end{array}\right.
$$

This is not a satisfactory result, because there is no general connection between the signs of det $\left\|J_{(a b)}\right\|$ and det $\left\|J_{a b}\right\|$, while invertibility of the force-stretch relations requires that $\operatorname{det}\left\|J_{a b}\right\| \neq 0$, so that from (4.23) we have no information as to whether or not the IFS-condition holds. However, a little more can be learned from (4.23). Since $\alpha^{2}+\beta^{2} \geqq 2 \alpha \beta$, we see from (4.23) that

$$
\left\|J_{a b}\right\|>0 \Rightarrow \frac{\partial T_{a}}{\partial v_{b}} \frac{\partial T_{b}}{\partial v_{a}}<\frac{\partial T_{a}}{\partial v_{a}} \frac{\partial T_{b}}{\partial v_{b}}, \quad a \neq b .
$$

This is a reciprocal inequality, asserting that the product of transverse tangent moduli can never be as great as the product of the corresponding tangent moduli of extension. In particular, the lesser transverse modulus can never be so large as the greater modulus of extension.

More can be inferred by considering certain identities relating any $3 \times 3$ matrix $\boldsymbol{A}$ to its symmetric part, $\boldsymbol{S}$. Since

$$
\begin{aligned}
& A_{k m}=S_{k m}+e_{k m p} B^{p}, \\
& S_{k m} \equiv A_{(k m)}, \quad B^{p} \equiv \frac{1}{2} e^{p q r} A_{[q r]},
\end{aligned}
$$

writing $A \equiv \operatorname{det}\left\|A_{k m}\right\|, S \equiv \operatorname{det}\left\|S_{k m}\right\|$, we see that ${ }^{5}$

$$
\begin{aligned}
A_{k k} & =S_{k k}, \\
A\left(A^{-1}\right)^{k k} & =S\left(S^{-1}\right)^{k k}+\left(B^{k}\right)^{2}, \\
A & =S+S_{k m} B^{k} B^{m} .
\end{aligned}
$$

A matrix $\boldsymbol{A}$ is positive-definite if and only if its symmetric part, $\boldsymbol{S}$, is positivedefinite. If $S$ is positive-definite, the right-hand sides of (4.26) are positive. Thus if a $3 \times 3$ matrix $\boldsymbol{A}$ is positive-definite, its principal minors of orders 1, 2 , and 3 are positive, although the converse does not hold, in general, unless $\boldsymbol{A}$ is symmetric. Applying to $\left\|J_{a b}\right\|$ this simple but apparently not hitherto noticed theorem on $3 \times 3$ matrices, we infer at once that

$$
\left\|J_{a b}\right\|>0 \Rightarrow C \& \mathrm{~T}-\mathrm{E}^{+} \& \mathrm{IFS}^{+} \& \mathrm{E}-\mathrm{T}^{+} .
$$

(Again existence of a natural state is necessary for $C$ to be included on the righthand side, but not for the other conclusions.) However, from (4.26) we see that unless $J_{a b}=J_{b a}$, it is not generally possible to reverse the implication, nor have we been able to show that O-F and P-C follow from $\left\|J_{a b}\right\|>0$. Results as complete as (4.16) and (4.20) seem not to hold unless there is a stored-energy function.

With this much information about isotropic materials at our disposal, we are now in a position to consider elastic materials in the broadest sense.

5 More generally,

$$
A\left(A^{-1}\right)^{k m}=S\left(S^{-1}\right)^{k m}+e^{k m p} S_{p q} B^{q}+B^{k} B^{m} .
$$


We have given evidence favoring inequalities to be imposed upon principal forces $T_{a}$ rather than on principal stresses $t_{a}$. Of course, mathematically, one could set up and study in the same way inequalities restricting the stress-stretch relations $(2.15)_{1}$ rather than the force-stretch relations $(2.15)_{2}$. Let us denote by ISS the condition that the stress-stretch relations $(2.15)_{1}$, rather than the force-stretch relations $(2.15)_{2}$, be invertible. Then by simple proofs analogous to those given above in connection with (4.18) we see that

$$
\sum_{a=1}^{3}\left(\bar{t}_{i}-t_{a}\right)\left(\bar{v}_{a}-v_{a}\right)>0 \Rightarrow\left\{\begin{array}{l}
\text { ISS \& } \\
\text { T-E \& } \\
\text { E-T \& } \\
\text { B-E. }
\end{array}\right.
$$

If a natural state is assumed to exist, we may add (3.2) to the list on the righthand side, and of course the $\mathrm{C}$-inequalities are included as a special case. If we set

$$
j_{a b} \equiv \frac{\partial t_{a}}{\partial v_{b}},
$$

we may seek conditions that $\left\|j_{a b}\right\|>0$, but the results are not satisfying, since even for hyperelastic materials the matrix $\left\|i_{a b}\right\|$ is generally not symmetric, so that its definiteness does not give such full information about its entries as does the definiteness of $\left\|J_{a b}\right\|$. Of course we can derive analogues of (4.23), (4.24), and (4.27), viz

$$
\begin{aligned}
\left\|j_{a b}\right\|>0 \Leftrightarrow\left\{\begin{array}{l}
\mathrm{T}-\mathrm{E}^{+} \& \\
\left(\frac{\partial t_{a}}{\partial v_{b}}+\frac{\partial t_{b}}{\partial v_{a}}\right)^{2}<4 \frac{\partial t_{a}}{\partial v_{a}} \frac{\partial t_{b}}{\partial v_{b}} \& \\
\operatorname{det}\left\|j_{(a b)}\right\|>0 .
\end{array}\right. \\
\left\|j_{a b}\right\|>0 \Rightarrow\left\{\begin{array}{l}
\mathrm{C}-\mathrm{E}^{+} \& \\
\mathrm{IS} S^{+} \& \\
\mathrm{E}-\mathrm{T}^{+} \& \\
\frac{\partial t_{a}}{\partial v_{b}} \frac{\partial t_{b}}{\partial v_{a}}<\frac{\partial t_{a}}{\partial v_{a}} \frac{\partial t_{b}}{\partial v_{b}}, \quad a \neq b,
\end{array}\right.
\end{aligned}
$$

where $\mathrm{ISS}^{+}$stands for the condition $\operatorname{det}\left\|j_{a b}\right\| \neq 0$. These difficulties account, in our opinion, for the failure of BARTA ${ }^{3}$ to obtain a satisfactory set of static inequalities. BARTA assumed as plausible the following conditions:

$$
\begin{aligned}
& \text { 1. ISS } \\
& \text { 2. } \mathrm{E}-\mathrm{T}^{+} \\
& \text {3. } \frac{\partial v_{a}}{\partial t_{b}}<0 \text { if } a \neq b \\
& \text { 4. } \frac{\partial}{\partial t_{a}}\left(v_{1} v_{2} v_{3}\right)>0 .
\end{aligned}
$$

This last inequality, which asserts that increase of any principal stress always results in. increase of volume, he replaced by its infinitesimal approximation?

$$
\frac{\partial}{\partial t_{a}}\left(v_{1}+v_{2}+v_{3}\right)>0 \text {. }
$$

From (4.31) $)_{1,2,3}$ and (4.32) he inferred that $\left\|j_{a b}^{-1}\right\|>0$, and hence $\left\|j_{a b}\right\|>0$. From (4.30) we see that the converse need not be true. In evidence favoring principal forces,

B $[1957,1]$.

7 It is unnecessary to use this approximation, as BARTa does, in order to infer from his original assumptions that any surface element parallel to an axis of increased tension is also increased in area, since

$$
\frac{\partial \log \left(v_{1} v_{2}\right)}{\partial t_{1}}=\frac{\partial \log \left(v_{1} v_{2} v_{3}\right)}{\partial t_{1}}-\frac{\partial \log v_{2}}{\partial t_{1}}>0,
$$

directly from his assumptions 3 and 4 . 
rather than principal stresses, as natural variables for simple requirements of inequality in the finite theory, to the fragmentary nature of these results and the inappropriateness of any approximation such as (4.32) in the finite theory may be added the fact that neither ISS nor B-E holds for elastic fluids. Against BARTA's specific assumptions 3 and 4 may be brought also the charge that they lack any evident use in other contexts such as uniqueness and wave propagation.

\section{The Coleman-Noll and GCN conditions}

For hyperelastic materials of arbitrary symmetry Coleman \& NoLL ${ }^{1}$ proposed the following condition of restricted convexity:

$$
\hat{\Sigma}\left(\bar{x}^{k}, \alpha\right)-\hat{\Sigma}\left(x^{k}, \alpha\right)-\left(\bar{x}^{k}, \alpha-x^{k}, \alpha\right) \frac{\partial \hat{\Sigma}}{\partial x^{k}, \alpha}>0
$$

for each $x^{k}{ }_{, \alpha}$ and for every $\bar{x}^{k}{ }_{, \alpha}$ of the form

$$
\bar{x}_{, \alpha}^{k}=G_{m}^{k} x^{m}, \alpha,
$$

where $\boldsymbol{G}$ is a symmetric, positive-definite tensor other than 1 . This condition will be called the $C-N$ condition. Here $\hat{\Sigma}\left(x^{k}, \alpha\right)=\Sigma(C)$, the stored-energy function of the hyperelastic material.

Reversing the roles of $\bar{x}^{k}{ }_{\alpha \alpha}$ and $x^{k}{ }_{\alpha \alpha}$ in (5.1), and then subtracting the result from (5.1), we find that

$$
\left(\bar{x}^{k}, \alpha-x^{k}, \alpha\right)\left(\bar{T}_{k}^{\alpha}-T_{k}^{\dot{\alpha}}\right)>0
$$

for all pairs $x^{k},{ }_{\alpha}, \bar{x}^{k}, \alpha$, such that (5.2) holds and $\boldsymbol{G} \neq 1$. Here we have used the stress-strain relation (2.4) in the equivalent form

$$
T_{k}^{\alpha}=\frac{\partial \hat{\Sigma}\left(x^{m}, \beta\right)}{\partial x^{k}, \alpha}, \quad \bar{T}_{k}^{\alpha}=\frac{\partial \hat{\Sigma}\left(\bar{x}^{m}, \beta\right)}{\partial \bar{x}^{k}, \alpha} .
$$

Inequality (5.3) is equivalent to inequality (5.1) if the domain of $\hat{\Sigma}\left(x^{k}, a\right)$ is convex. This can be seen as follows. First note that

$$
\hat{\Sigma}\left(\bar{x}^{k}, \alpha\right)-\hat{\Sigma}\left(x^{k}, \alpha\right)=\int_{x^{k}, \alpha}^{\bar{z}^{k}, \alpha} \frac{\partial \Sigma}{\partial \tilde{x}^{m}, \beta} d \tilde{x}^{m}, \beta,
$$

where the path of integration in the 9-dimensional space of $\tilde{x}_{, \beta}^{m}$ is any curve joining $x^{k}{ }_{\alpha}$ to $\bar{x}^{k}, \alpha$, within the domain of definition of $\hat{\Sigma}$. If that domain is convex, we may choose as the path of integration the straight line

$$
\tilde{x}^{k}{ }_{\alpha}=x^{k}{ }_{\alpha}+\lambda\left(\bar{x}^{k}{ }_{\alpha}-x^{k}{ }_{, \alpha}\right), \quad 0 \leqq \lambda \leqq 1 .
$$

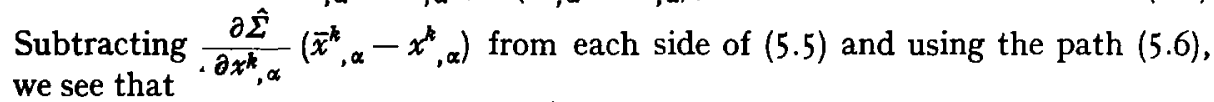

$$
\begin{aligned}
& \hat{\Sigma}\left(\bar{x}^{k}, \alpha\right)-\hat{\Sigma}\left(x^{k}, \alpha\right)-\frac{\partial \hat{\Sigma}}{\partial x^{k}, \alpha}\left(\bar{x}^{k}, \alpha-x^{k}{ }_{, \alpha}\right) \\
& =\int_{0}^{1} \frac{\partial \hat{\Sigma}}{\partial \tilde{x}^{k}, \alpha}\left(\tilde{x}^{k}, \alpha-x^{k}, \alpha\right) \frac{d \lambda}{\lambda}-\frac{\partial \hat{\Sigma}}{\partial x^{k}, \alpha}\left(\bar{x}^{k}, \alpha-x^{k}{ }_{, \alpha}\right), \\
& =\int_{0}^{1}\left(\frac{\partial \Sigma}{\partial \tilde{x}^{k}, \alpha}-\frac{\partial \Sigma}{\partial x^{k}, \alpha}\right)\left(\tilde{x}^{k}, \alpha-x^{k}, \alpha\right) \frac{d \lambda}{\lambda} \text {. } \\
& =\int_{0}^{1}\left(\widetilde{T}_{k}^{\alpha}-T_{k}^{\alpha}\right)\left(\tilde{x}_{, \alpha}^{k}-x^{k}, \alpha\right) \frac{d \lambda}{\lambda} \text {. }
\end{aligned}
$$

${ }^{1}[1959, \S 8]$. 


$$
\tilde{x}_{, \alpha}^{k}=\left[(1-\lambda) \delta_{m}^{k}+\lambda G_{m}^{k}\right] x^{m}, \alpha,
$$

the tensor in brackets being symmetric and positive-definite when $0 \leqq \lambda \leqq 1$, the inequality (5.3) holds at every point on the path of integration, so that the integrand is positive, and (5.1) follows.

It has been shown, then, that (5.1) and (5.3) are equivalent, locally, for hyperelastic materials. For more general elastic materials, however, $(5.3)$ is a meaningful condition, which we may justly call the generalized Coleman-Noll condition, or GCN condition.

Let us apply the GCN condition to the classical linearized theory. Choosing $\bar{x}^{k}{ }_{\alpha}=g_{\alpha}^{k}$, so that $\bar{T}_{k}^{\alpha}=0$ (assuming, as usual in the classical theory, that there is a natural state), while $x^{k}{ }_{\alpha} \approx g_{\alpha}^{k}+E^{k}{ }_{\alpha}$, where $E^{k}{ }_{\alpha}$ represents an infinitesimal strain, from (5.3) we infer that $t^{k m} E_{k m}>0$ if not all the $E_{k m}=0$. That is, the work done in any non-rigid infinitesimal deformation is positive. Therefore, for materials of arbitrary symmetry,

$\mathrm{GCN} \Rightarrow$ all conditions customarily imposed in the linearized theory ${ }^{2}$.

In other words, the GCN condition implies that the beginning of any process of deformation from the natural state will require work to be done, although in finite strain there remains the possibility of certain workless deformations. Thus the GCN condition may not rule out a certain kind of energetic instability in large strain.

The proof of uniqueness given in connection with the $\mathrm{GCN}_{0}$ condition can now be generalized. Assume that two deformation gradients, $\bar{x}^{k}{ }_{, \alpha}$ and $x^{k}{ }_{, \alpha}$, related by a non-identical pure stretch, can lead to the same Piola-Kirchhoff stress tensor, so that $\bar{T}_{k}^{\alpha}=T_{k}^{\alpha}$. From (5.3) a contradiction follows. That is, if we write (2.1) in the form $\boldsymbol{T}=\mathfrak{h}(\boldsymbol{F})$, where $\boldsymbol{F}$ stands for $x^{m}, \beta$, then, for any fixed $\boldsymbol{F}$,

$$
\mathfrak{h}(\boldsymbol{G} \boldsymbol{F}) \neq \mathfrak{h}(\boldsymbol{F})
$$

for any positive-definite, symmetric $\boldsymbol{G}$ other than 1 . In particular, by choosing $\boldsymbol{F}=\mathbf{1}$ we infer that a unique value of $\boldsymbol{T}$ corresponds to an assigned pure strain from the reference configuration. More generally,

$$
\mathrm{GCN} \Rightarrow \text { invertibility of the stress-strain relations in pure strain, }
$$

as indicated above, although it certainly does not suffice for a unique Cauchy stress $t$ to be determined by a given strain and rotation ${ }^{3}$.

$2 \mathrm{By}$ "the linearized theory" we mean here the theory of $\mathrm{CAUCHY}_{\mathrm{A}}$, in which there need be no stored-energy function. Contrary to widespread misconception, the requirement that work must be done in every infinitesimal deformation does not imply that there is a stored-energy function. The condition of positive work demands that $\mathfrak{I}^{k m p q} E_{k m} E_{p q}>0$ for every symmetric, non-vanishing $\boldsymbol{E}$. This requirement restricts the symmetric part of the tensor of linear elasticities, namely, $\frac{1}{2}\left(l^{k m p q}+\mathfrak{l}^{p q k m}\right)$, leaving the skew-symmetric part, namely, $\frac{1}{2}\left(1^{k m p q}-\prod^{p q k m}\right)$, entirely unrestricted since it contributes nothing to the work done in any deformation. For there to be a storedenergy function, it is necessary and sufficient that $\Upsilon^{k m q p}=\mathfrak{l}^{p q k m}$.

${ }^{3}$ If it did, we should infer that $\mathrm{GCN}_{0} \Rightarrow \operatorname{det}\left\|j_{a b}\right\|>0$ for isotropic materials, and we know this is not always true. For example, in an elastic fluid det $\left\|j_{a b}\right\|=0$, but the $\mathrm{GCN}_{0}$ condition may be satisfied. More generally, (5.10) does not imply, in general, that the equation $\boldsymbol{T}=\boldsymbol{G}(\boldsymbol{G})$ is invertible for $\boldsymbol{G}$ when $\boldsymbol{T}$ and $\boldsymbol{F}$ are given. 
For the special case of isotropic materials, in a principal co-ordinate system $\boldsymbol{T}=\operatorname{diag}\left(T_{1}, T_{2}, T_{3}\right) \boldsymbol{R}$ and $x^{m}{ }_{, \beta}=\operatorname{diag}\left(v_{1}, v_{2}, v_{3}\right) \boldsymbol{R}$, where $\boldsymbol{R}$ is a rotation matrix. To apply (5.3), we take $\bar{x}^{m}{ }_{, \beta}=\boldsymbol{G} \operatorname{diag}\left(v_{1}, v_{2}, v_{3}\right) \boldsymbol{R}$. In the special case when $\boldsymbol{G}$ has the same principal axes as does the original strain, $\bar{x}^{m}{ }_{, \beta}=\operatorname{diag}\left(\bar{v}_{1}, \bar{v}_{2}, \bar{v}_{3}\right) \boldsymbol{R}$, and in this special case (5.3) reduces to (4.18). That is, for isotropic materials,

$$
\mathrm{GCN} \Rightarrow \mathrm{GCN}_{0} \text {. }
$$

Therefore, by (4.23), the GCN condition implies all the static inequalities laid down as plausible for isotropic materials.

Can the implication be reversed? That is, is $\mathrm{GCN}_{0}$ equivalent to $\mathrm{GCN}$ for isotropic materials? In a narrower sense the question was raised by COLEMAN $\&$ NOLL $^{4}$, who showed that for isotropic hyperelastic materials

$$
\mathrm{C}-\mathrm{N} \Rightarrow \tilde{\Sigma}() \text { is convex, }
$$

and they asked if, conversely, convexity of $\tilde{\Sigma}()$ implies the C-N condition. BraGg \& Coleman ${ }^{5}$ constructed a counter-example showing that the answer is no. It follows a fortiori that

$$
\mathrm{GCN}_{\mathbf{0}} \Rightarrow \mathrm{GCN} \text {. }
$$

\section{The $\mathrm{C}-\mathrm{N}^{+}$and $\mathrm{GCN}^{+}$conditions}

We now recall the connection between the convexity of a function and the definiteness of its Hessian form. If a twice continuously differentiable function $F\left(x^{1}, x^{2}, \ldots, x^{N}\right)$ of $N$ variables $x^{\Gamma}, \Gamma=1,2, \ldots, N$, satisfies the inequality

$$
F(\bar{x})-F(\boldsymbol{x})-\sum_{\Gamma=1}^{N}\left(\bar{x}^{\Gamma}-x^{\Gamma}\right) \frac{\partial F(\boldsymbol{x})}{\partial x^{\Gamma}}>0
$$

for all $\overline{\boldsymbol{x}}$ other than $\boldsymbol{x}$ itself, i.e., if the surface $F(\boldsymbol{x})=0$ is (strictly) convex at $\boldsymbol{x}$, then the Hessian matrix is positive-semidefinite at $\boldsymbol{x}$ :

$$
\left\|\frac{\partial^{2} F}{\partial x^{\Gamma} \partial x^{4}}\right\| \geqq 0
$$

while a sufficient condition for (6.1) to hold is

$$
\left\|\frac{\partial^{2} F}{\partial x^{\Gamma} \partial x^{\Delta}}\right\|>0
$$

in the notation already used to denote a positive-definite matrix. Moreover, it has been shown ${ }^{1}$ that if $F()$ is convex at every point $x$ in an open set, then the condition (6.3) can fail at most on a nowhere dense set. At a point where (6.3) holds, we may say that $F$ is strongly convex.

Similar results hold for the more general inequality

$$
\sum_{\Gamma=1}^{N}\left(\bar{x}^{\Gamma}-x^{\Gamma}\right)\left(F_{\Gamma}(\overline{\boldsymbol{x}})-F_{\Gamma}(\boldsymbol{x})\right)>0
$$

for all $\overline{\boldsymbol{x}}$ other than $\boldsymbol{x}$. Here it is $N$ functions $F_{\Gamma}$, not necessarily the derivatives of a single function, that are restricted. We can see easily that a sufficient

[1959, §12].

5 [1963].

1 Bernstein \& Toupin [1962, 1, Theorem VI]. 
condition for (6.4) to hold is that the Jacobian matrix be positive-definite:

$$
\left\|\frac{\partial F_{\Gamma}(\boldsymbol{x})}{\partial x^{\Delta}}\right\|>0
$$

or, equivalently, its symmetric part shall be positive-definite, while a necessary condition is that the Jacobian matrix be positive semi-definite. If a transformation $\boldsymbol{x} \rightarrow \boldsymbol{F}(\boldsymbol{x})$ satisfies (6.4), we shall call it a convex transformation, and if it satisfies the stronger condition (6.5), we shall call it a strongly convex transformation. If $\partial F_{\Gamma} / \partial x^{\Delta}=\partial F_{\Delta} / \partial x^{\Gamma}$, then there exists a function $F()$ such that $F_{\Gamma}=\partial F / \partial x^{r}$, and if the transformation $\boldsymbol{x} \rightarrow \boldsymbol{F}$ is convex, then $F()$ is a convex function, and vice versa, etc. For example, the $\mathrm{GCN}_{0}$ inequality (4.18) asserts that the transformation from principal stretches to principal forces is a convex transformation; in consequence the stored-energy function, if it exists, is a convex function of the principal stretches.

Returning to the $\mathrm{C}-\mathrm{N}$ and GCN conditions, we see that the essential restriction $\bar{x}^{k}, \alpha=G_{m}^{k} x^{m}{ }_{, \alpha}$ where $G$ is symmetric and positive-definite, prevents immediate application of the foregoing results concerning Jacobian and Hessian matrices. Rather, we define $F_{k}^{\alpha}$ as a function of the two tensor variables $G_{p}^{m}$ and $x_{, \beta}^{q}$ as ollows:

$$
F_{k}^{\alpha}\left(G_{p}^{m}, x^{q}, \beta\right) \equiv \mathfrak{h}_{k}^{\alpha}\left(G_{p}^{m} x^{p}, \beta\right),
$$

so that, in particular, $F_{k}^{\alpha}\left(\delta_{p}^{m}, x^{q}{ }_{\beta}\right)=T_{k}^{\alpha}$, by (5.9). Then the GCN-condition (5.3) is equivalent to

$$
\left(G_{m}^{k}-\delta_{m}^{k}\right) x^{m}, \alpha\left(F_{k}^{\alpha}\left(G_{p}^{m}, x^{q}{ }_{, \beta}\right)-F_{k}^{\alpha}\left(\delta_{p}^{m}, x^{\dot{q}}{ }_{\beta}\right)\right)>0,
$$

for each fixed $x^{k}{ }_{\alpha}$, and for all $\boldsymbol{G}$ other than the identity. Comparison with (6.4) shows that for each fixed $x^{m}{ }_{, \alpha}$, the transformation $G_{q}^{p} \rightarrow x^{m}{ }_{, \alpha} F_{k}^{\alpha}$ is a convex transformation in every neighborhood of the point $\boldsymbol{G}=\mathbf{1}$ in the 6-dimensional space of symmetric tensors. The corresponding Jacobian matrix is

$$
\begin{aligned}
\left.\frac{\partial}{\partial G_{q}^{p}}\left(x^{m}{ }_{, \alpha} F_{k}^{\alpha}\left(G_{q}^{p}, x^{\gamma}, \beta\right)\right)\right|_{\boldsymbol{G}=1} & =x^{m},\left.\alpha \frac{\partial \mathfrak{h}_{k}{ }^{\alpha}\left(G_{s}^{r} x^{s}, \beta\right)}{\partial G_{q}^{p}}\right|_{\boldsymbol{G}=1}, \\
& =x^{m}{ }_{, \alpha} \frac{\partial T_{k}^{\alpha}}{\partial x^{\gamma}, \beta} \delta_{p}^{\gamma} \delta_{s}^{q} x^{s}, \beta=\frac{\varrho_{0}}{\varrho} B_{k}{ }^{m}{ }_{p}^{q},
\end{aligned}
$$

by (5.9) and (2.9). Accordingly, $B^{k m p q} G_{k m} G_{p q} \geqq 0$ for all symmetric $\boldsymbol{G}$, or, equivalently,

$$
B^{(k m)(p q)} h_{k m} h_{p q} \geqq 0
$$

for every $\boldsymbol{h}$. Let us call the corresponding condition of strong convexity, viz

$$
B^{(k m)(p q)} h_{k m} h_{p q}>0 \text { for every non-vanishing } \boldsymbol{h} \text {, }
$$

the $\mathrm{GCN}^{+}$condition ${ }^{2}$, or, when there is a stored-energy function, simply the $C-N^{+}$condition. Conversely, if (6.10) holds for each $x^{k}{ }_{\alpha}$ in a region, from (6.8) it follows that, a fortiori, the Jacobian matrix calculated for an arbitrary $\boldsymbol{G}$ sufficiently near to 1 is positive-definite for each fixed $x^{k}, \alpha$. Hence the transformation $G_{q}^{b} \rightarrow x^{m}{ }_{\alpha} F_{k}^{\alpha}$ is convex in a neighborhood of $\boldsymbol{G}=\mathbf{1}$. It has been shown, then, that

$$
\mathrm{GCN}^{+} \Rightarrow \mathrm{GCN} \text {, and } \mathrm{C}-\mathrm{N}^{+} \Rightarrow \mathrm{C}-\mathrm{N} \text {. }
$$

These implications cannot be reversed.

${ }^{2}$ For hyperelastic materials (6.9) was derived from the $\mathrm{C}-\mathrm{N}$ condition by ToupiN \& BeRnsteIN [1961, 5, §3]. They determined some consequences of the stronger condition (6.10), which was then set up for general elastic materials by TRUESDELL [1961, 6, Eq. (10.4)], who found its implications upon wave propagation. 
For isotropic materials we can put the $\mathrm{GCN}^{+}$-condition into an immediately plausible static form. The condition (6.10) asserts that a certain $6 \times 6$ matrix $\boldsymbol{M}$, having as components the 36 possibly distinct components of $B^{(k m)(p q)}$, is positivedefinite. For an isotropic material, in a principal co-ordinate system we may calculate this matrix $\boldsymbol{M}$ explicitly by aid of (2.11) and (2.12), finding that

where

$$
\boldsymbol{M}=\left\|\begin{array}{cccccc}
\frac{v_{1}}{v_{2} v_{3}} \frac{\partial T_{1}}{\partial v_{1}} & \frac{1}{v_{3}} \frac{\partial T_{1}}{\partial v_{2}} & -\frac{1}{v_{2}} \frac{\partial T_{1}}{\partial v_{3}} & 0 & 0 & 0 \\
\frac{1}{v_{3}} \frac{\partial T_{2}}{\partial v_{1}} & \frac{v_{2}}{v_{1} v_{3}} \frac{\partial T_{2}}{\partial v_{2}} & -\frac{1}{v_{1}} \partial T_{2} & 0 & 0 & 0 \\
\frac{1}{v_{2}} \cdot \frac{\partial T_{3}}{\partial v_{1}} & \frac{1}{v_{1}} \frac{\partial T_{3}}{\partial v_{2}} & -\frac{v_{3}}{v_{1}} \frac{\partial T_{3}}{\partial v_{3}} & 0 & 0 & 0 \\
0 & 0 & 0 & A_{1} & 0 & 0 \\
0 & 0 & 0 & 0 & A_{2} & 0 \\
0 & 0 & 0 & 0 & 0 & A_{3}
\end{array}\right\|
$$

$$
A_{1}=\frac{\left(t_{2}-t_{3}\right)\left(v_{2}^{2}+v_{3}^{2}\right)}{2\left(v_{2}^{2}-v_{8}^{2}\right)}-\frac{1}{4}\left(t_{2}+t_{3}\right) \text {, }
$$

etc. The $3 \times 3$ matrix in the upper left-hand corner is proportional to $v_{a} v_{b} J_{a b}$ and hence is positive-definite if and only if $\left\|J_{a b}\right\|>0$. Thus

$$
\mathrm{GCN}^{+} \Leftrightarrow\left(\left\|J_{a b}\right\|>0\right) \&\left(A_{\Gamma}>0\right) \text {. }
$$

In $\S 4$ we have analysed the condition $\left\|J_{a b}\right\|>0$. It is natural to ask if the condition $A_{\Gamma}>0$ follows from it. L. BRAGG has shown us a proof that such is not the case. Thus a full understanding of the $\mathrm{GCN}^{+}$condition would require statical interpretation of the inequalities $A_{\Gamma}>0$. While we are not able to construct such an interpretation in general, we can go some way toward it. Let the principal stretches be ordered by number: $v_{1}>v_{2}>v_{3}$. If $f(r)$ is the function defined by (4.5), and if $g(r) \equiv f(r) / r=\left(r^{2}+3\right) /\left(1+3 r^{2}\right)$, then directly from (6.13) and $(2.13)$ we see that

$$
\begin{aligned}
& A_{3}>0 \Leftrightarrow f\left(\frac{v_{1}}{v_{2}}\right) T_{1}>T_{2} \Leftrightarrow g\left(\frac{v_{1}}{v_{2}}\right) t_{1}>t_{2}, \\
& A_{1}>0 \Leftrightarrow f\left(\frac{v_{2}}{v_{3}}\right) T_{2}>T_{3} \Leftrightarrow g\left(\begin{array}{c}
v_{2} \\
v_{3}
\end{array}\right) t_{2}>t_{3}, \\
& A_{2}>0 \Leftrightarrow T_{1}>f\left(\begin{array}{c}
v_{3} \\
v_{1}
\end{array}\right) T_{3} \Leftrightarrow t_{1}>g\left(\begin{array}{c}
v_{3} \\
v_{1}
\end{array}\right) t_{3} .
\end{aligned}
$$

In $\S 4$ we showed that $f(r)>1$ if $r>1$ and that $0<f(r)<1$ if $0<r<1$. Accordingly, if the $\mathrm{O}-\mathrm{F}$ inequalities hold and if $T_{1} \geqq 0, T_{2} \geqq 0$, we infer from (6.15) that $A_{\Gamma}>0, \Gamma=1,2,3$. Since $0<g(r)<1$ if $r>1$ but $g(r)>1$ if $0<r<1$, from (6.15) we infer ${ }^{3}$ also that if the B-E inequalities hold, and if $t_{3} \leqq 0, t_{2} \leqq 0$, then $A_{I}>0, \Gamma=1,2,3$. Therefore, if $v_{1}>v_{2}>v_{3}$,

or

$$
\left.\begin{array}{l}
\text { O-F } \&\left(t_{1} \geqq 0\right) \&\left(t_{2} \geqq 0\right) \\
\text { B-E } \&\left(t_{3} \leqq 0\right) \&\left(t_{2} \leqq 0\right)
\end{array}\right\} \Rightarrow A_{\Gamma}>0 \text {. }
$$

${ }^{3}$ In detail, we multiply the inequality $g\left(v_{3} / v_{1}\right)>1$ by the inequality $-t_{3}>-t_{1}$, recalling that $-t_{3} \geqq 0$. By $(6.15)_{2}$, then, $A_{2}>0$. Next we multiply the inequality $1>g\left(v_{2} / v_{3}\right)$, by the inequality $-t_{3}>-t_{2}$. Since $-t_{3} \geqq 0$, by $(6.15)_{0}$ it follows that $A_{1}>0$. A parallel argument, using the assumption that $-t_{2} \geqq 0$, shows that $A_{3}>0$. 
If we now assume that both the O-F and B-E inequalities hold, we see that no states of stress are excluded by the further assumptions listed in (6.16). Indeed, if $t_{2}<0$, by the B-E inequalities it follows that $t_{3}<0$. Likewise, if $t_{2}>0$, by the $\mathrm{O}-\mathrm{F}$ inequalities it follows that $t_{1}>0$. Thus we have shown that

$$
\mathrm{O}-\mathrm{F} \& \mathrm{~B}-\mathrm{E} \Rightarrow A_{\boldsymbol{\Gamma}}>0 \text {. }
$$

The implication cannot be reversed. From (6.14) and (6.17) it follows that

$$
\left(\left\|J_{a b}\right\|>0\right) \& \mathrm{O}-\mathrm{F} \& \mathrm{~B}-\mathrm{E} \Rightarrow \mathrm{GCN}^{+} \text {, }
$$

where again the inequality cannot be reversed.

Returning to the complete result (6.14), we remark that the formal conditions $\left\|J_{a b}\right\|>0$ and $A_{\Gamma}>0$ deserve notice, since they afford an immediate and simple test for finding whether or not any given stress-strain relation for isotropic materials satisfies the $\mathrm{GCN}^{+}$condition. The two parts of the test refer to different aspects of the stress-strain relations. The condition $\left\|J_{a b}\right\|>0$, asserting that the transformation from principal stretches to principal forces is strongly convex, expresses a comparison of the forms of the stress-strain relations at pairs of points, while the independent conditions $A_{r}>0$ restrict those forms at a single point. To make this latter fact particularly clear, observe from (3.18) and (6.17) that

and hence by (6.18) that

$$
\mathrm{E} \Rightarrow A_{\Gamma}>0 \text {, }
$$

$$
\left(\left\|J_{a b}\right\|>0\right) \& \mathrm{E} \Rightarrow \mathrm{GCN}^{+} \text {. }
$$

It has already been mentioned that what empirical evidence there is seems to favor the E-inequalities (3.17), which are no more than statements of sign for the coefficients in a particular representation of the stress. In a case where these simple and immediate inequalities are satisfied, one has only to test the Jacobian matrix $\left\|J_{a b}\right\|$ in order to see if the $\mathrm{GCN}^{+}$condition is satisfied.

A different chain of implication may be formed from (6.11), (5.10), and (4.22):

$$
\mathrm{GCN}^{+} \Rightarrow \mathrm{GCN} \Rightarrow \mathrm{GCN}_{0} \Rightarrow\left\{\begin{array}{l}
\text { P-C \& } \\
\text { IFS \& } \\
\text { T-E \& } \\
\text { E-T \& } \\
\text { O-I. }
\end{array}\right.
$$

By use of (6.14) and (4.28) we may strengthen (6.21) a little ${ }^{4}$ :

$$
\mathrm{GCN}^{+} \Rightarrow\left\{\begin{array}{l}
\mathrm{P}-\mathrm{C} \& \\
\mathrm{IFS}^{+} \& \\
\mathrm{~T}-\mathrm{E}^{+} \& \\
\mathrm{E}-\mathrm{T}^{+} \& \\
\mathrm{O}-\mathrm{F} .
\end{array}\right.
$$

In view of the remark following (6.14), this implication cannot be reversed.

4 The implication $\mathrm{C}-\mathrm{N}^{+} \Rightarrow \mathrm{T}-\mathrm{E}^{+}$is equivalent to a theorem on wave speeds asserted by ToupIN in his Newcastle lecture of 1961 ; the more general result $\mathrm{GCN}^{+} \Rightarrow \mathrm{T}-\mathrm{E}^{+}$

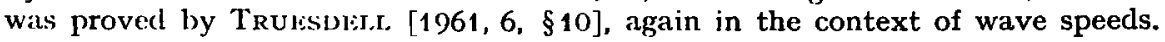


The results in this section up to now, including the major theorems schematized by $(6.14),(6.18),(6.21)$, and (6.22), are valid for all isotropic elastic media, whether or not there be a stored-energy function. (As usual, existence of a natural state is presumed so as to include the P-C inequality but is unnecessary for the others, in each case.)

For hyperelastic materials, some stronger results follow, since the condition $\left\|J_{a b}\right\|>0$ is equivalent to simple static inequalities. Thus (6.14) may now be replaced by

$$
\left.\begin{array}{lr}
\mathrm{C} & \& \\
\mathrm{IFS}^{+} & \& \\
\mathrm{~T}-\mathrm{E}^{+} & \& \\
\mathrm{E}-\mathrm{T}^{+} & \& \\
A_{\Gamma}>0 & 0
\end{array}\right\} \Leftrightarrow{\mathrm{C}-\mathrm{N}^{+}}^{+}
$$

This result cannot be weakened, since Brar:-r, \& ColEman ${ }^{5}$ have constructed a strongly convex stored-energy function that does not satisfy the $\mathrm{C}-\mathrm{N}^{+}$condition; that is, a function such that $\left\|J_{a b}\right\|>0$ but $A_{\Gamma}<0$ for some strains, despite the fact that the O-F inequalities hold. Since, by (4.20), $\left\|J_{a b}\right\|>0 \Rightarrow 0-\mathrm{F}$ for hyperelastic materials, the one-way implications (6.18) and (6.20) may be simplified a little:

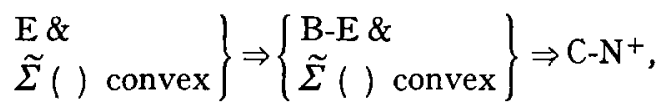

affording simple and immediate tests sufficient to ensure that the $\mathrm{C}-\mathrm{N}^{+}$condition holds.

The implications revealed in this section are the main results of our study. To us it seems particularly enlightening to have replaced the $\mathrm{C}-\mathrm{N}^{+}$condition by an equivalent requirement of five independent, simple, static inequalities, all but the last of which $\left(A_{\Gamma}>0\right)$ are immediately plausible. For the more general theory, (6.22) shows that the $\mathrm{GCN}^{+}$condition covers by its implications a full set of plausible static inequalities. That is, the static response of an isotropic elastic material satisfying the $\mathrm{GCN}^{+}$condition will not exhibit any phenomena contradicting the principles we have laid down as physically natural for static deformation. Those principles, however, do not compel us to adopt the $\mathrm{GCN}^{+}$ condition: other generalizations of the $\mathrm{C}^{-\mathrm{N}^{+}}$condition may serve just as well.

\section{The condition of strong ellipticity}

A simple invariant condition, meaningful for all elastic materials, is the requirement that the differential equations of equilibrium be strongly elliptic. This $S-E$ condition may be expressed in the form?

$$
B^{k p m q} l_{k} l_{m} L_{p} L_{q}>0
$$

for arbitrary non-vanishing vectors $\boldsymbol{l}$ and $\boldsymbol{L}$. While the main mechanical significance of this condition is undulatory, since for hyperelastic materials it is necessary and sufficient that corresponding to each direction of propagation 5 [1963].

1 Derived by Toupin \& Bernstein $[1961,5, \S 3]$ for hyperelastic materials and more generally by TRUESDELL $[1961,6$, Eq. (10.3)]. 
there exist at least $t$ ' ? real amplitudes with positive speeds of propagation, it is worthwhile to determine its consequences for statics. For the linearized theory of isotropic materials, (7.1) reduces to (1.2), weaker than (1.1) and hence too weak to ensure physically reasonable response in statics. On the other hand, although (1.1), and hence also the C-N condition, implies the S-E condition in the linearized theory, ToupIN \& BERNSTEIN ${ }^{2}$ have constructed an example in which, for a certain range of strains, the $\mathrm{C}-\mathrm{N}$ condition is satisfied but the S-E condition is not. Thus, generally,

$$
\mathrm{C}-\mathrm{N} \Rightarrow \mathrm{S}-\mathrm{E}, \quad \mathrm{S}-\mathrm{E} \neq \mathrm{C}-\mathrm{N},
$$

and in these statements of non-implication we may replace $\mathrm{C}-\mathrm{N}$ by C-N ${ }^{+}, \mathrm{GCN}$, $\mathrm{CGN}_{0}$, or $\mathrm{GCN}^{+}$; if we please. Although the results following from a condition of Coleman \& Noli's type must therefore be different in general from those following from the condition of strong ellipticity, nevertheless the two conditions do have certain consequences in common. For example, in hyperelastic materials the $\mathrm{C}-\mathrm{N}$ condition is sufficient that for any given direction of propagation there be at least one squared wave-speed that is positive, and for principal waves in isotropic materials this speed corresponds to the longitudinal waves ${ }^{3}$. We shall now see this latter fact and some others of a like kind in the present, purely static, context.

For isotropic elastic materials, substitution of (2.12) into (7.1) yields the inequality

$$
T_{p q}^{k m} B^{q s} l_{k} l^{p} L_{m} L_{s}>0
$$

for arbitrary non-vanishing vectors $\boldsymbol{l}$ and $\boldsymbol{L}$. Let $\boldsymbol{L}$ be a unit proper vector of $\boldsymbol{B}$, so that $B^{q s} L_{s}=v_{1}^{2} L^{q}$, say. Then (7.3) becomes

$$
T_{p q}^{k m} l_{k} l^{p} L_{m} L^{q}>0
$$

for all non-vanishing vectors $l$. In a principal co-ordinate system, this condition assumes the form

equivalently,

$$
T^{11}{ }_{11}^{2}+T_{12}^{12} l_{2}^{2}+T^{13}{ }_{13}^{2}>0 ;
$$

$$
T^{11}{ }_{11}>0, \quad T^{12}{ }_{12}>0, \quad T^{13}{ }_{13}>0 .
$$

From (2.11) we see that (7.6) is equivalent to the $\mathrm{T}-\mathrm{E}^{+}$and B-E inequalities. That is, for isotropic materials ${ }^{4}$

$$
\mathrm{S}-\mathrm{E} \Rightarrow \mathrm{T}-\mathrm{E}^{+} \& \mathrm{~B}-\mathrm{E} \text {. }
$$

While $\mathrm{T}-\mathrm{E}^{+} \& \mathrm{~B}-\mathrm{E}$ imply (7.4), they do not imply the S-E condition, since (7.4) is the special case arising when $\boldsymbol{L}$ is a proper vector of $\boldsymbol{B}$. As $S-E=\Rightarrow C$, it follows that

$$
\mathrm{S}-\mathrm{E} \Rightarrow \mathrm{P}-\mathrm{C} \text { or IFS, }
$$

${ }^{2}[1961,5, \S 3]$. Alternatively, we may note that the $\mathrm{C}-\mathrm{N}$ condition may be satisfied by an elastic fluid, but the S-E condition never can be.

${ }^{3}$ TRuesdell $[1961,6, \$ \S 10,12]$.

4 The implication S-E $\Rightarrow$ B-E was first derived, apparently, by HAYEs \& RIVLIN $[1961,3]$. They considered only hyperelastic materials. TRUESUELL $[1956,2]$ remarked that ERICKSEN \& ToupIN had shown him a proof that S-E $\Rightarrow$ T-E for hyperelastic materials. 
since the C-inequalities are necessary in order that either of the inequalities on the right-hand side of (7.8) may hold.

While the difference in nature between the S-E and $\mathrm{C}-\mathrm{N}$ conditions is sufficiently revealed by comparing (7.7) and (7.8) with (6.23), it would be worthwhile to find a complete statical interpretation for the S-E condition in isotropic materials. Although it is easy to write out the form assumed by the S-E condition in a principal co-ordinate system, we have been unable to interpret the result ${ }^{5}$.

It is interesting to contrast the two main conditions when they are expressed in terms of a common quadratic form:

$$
B^{k m p q} h_{k m} h_{p q}>0 \text {,. }
$$

where it is only the domain of non-vanishing tensors $\boldsymbol{h}$ that differs in the two cases, namely,

$$
\text { GCN }{ }^{r} \text { : all symmetric } \boldsymbol{h}
$$

S-E: all $\boldsymbol{h}$ of rank 1 .

The presence or absence of a stored-energy function seems not to affect these comparisons in any way.

\section{Three uniqueness theorems}

As was pointed out by HILL ${ }^{1}$, the formal steps used to derive KIRCHHOFF's classical uniqueness theorem may be followed also in the finite theory. Since the equations of equilibrium may be written in the form ${ }^{2}$

$$
T_{k}^{\alpha} ; \alpha+\varrho_{0} t_{k}=0 \text {, }
$$

$f_{k}$ being the field of body force and $\varrho_{0}$ the density in the reference configuration, use of the divergence theorem leads at once to an identity of KIRCHHOFF's type:

$$
\begin{aligned}
\int_{\mathscr{B}_{0}}\left(\bar{T}_{k}^{\alpha}-T_{k}^{\alpha}\right)\left(\bar{x}_{, \alpha}^{k}-x^{k}, \alpha\right) d V_{0} \\
=\int_{\mathscr{S}_{0}} \varrho_{0}\left(\bar{f}_{k}-f_{k}\right)\left(\bar{u}^{k}-u^{k}\right) d V_{0}+\underset{\delta \mathscr{F}_{0}}{\oint}\left(\bar{u}^{k}-u^{k}\right)\left(\bar{T}_{k}^{\alpha}-T_{k}^{\alpha}\right) d S_{\alpha},
\end{aligned}
$$

where $\mathscr{B}_{0}$ is a region in the reference configuration and where $u^{k}$ is the displacement vector. In this identity a bar distinguishes quantities associated with a second deformation-gradient field $\bar{x}^{k}, \alpha$. Suppose now that there are two solutionpairs, $\left(x^{k}, \alpha, T_{m}^{\beta}\right)$ and $\left(\bar{x}^{k},{ }_{\alpha}, \bar{T}_{m}^{\beta}\right)$, to the same mixed boundary-value problem. Then $\bar{f}_{k}=f_{k}$ in $\mathscr{B}_{0}$, and on $\partial \mathscr{B}_{0}$ either $\bar{u}^{k}=u^{k}$ or $\bar{T}_{k}^{\alpha} d S_{\alpha}=T_{k}^{\alpha} d S_{\alpha}$. From (8.2)

${ }^{5}$ Likewise Zorski $[1962,6, \$ 2]$ gives up his attempt to interpret the S-E condition directly, resting content with consequences and special cases. An interpretation in terms of steady velocity ficlds; has been constructed by HiLL [1962, 5, \$4(i)].

$1[1957,3, \$ 2]$. HiLl, presuming the material to be hyperelastic, observed from the corresponding special case of (8.3) that convexity of $\hat{\Sigma}()$ in the 9-dimensional space of the $x^{k},{ }_{\alpha}$ would suffice to yield unqualified uniqueness of solution to the general mixed boundary-value problem and hence is too strong a restriction to be imposed on all hyperelastic materials for all states of strain. Below we consider his result in generalized form.

2 Apparatus sufficient to derive (8.1) and (8.2) is given $[1960,3, \S \S 19,210$, App. 20]. 
it follows that

$$
\int_{\mathscr{S}_{0}}\left(\bar{T}_{k}^{\alpha}-T_{k}^{\alpha}\right)\left(\bar{x}_{, \alpha}^{k}-x^{k}{ }_{, \alpha}\right) d V_{0}=0 .
$$

This condition may be satisfied in several ways: (1) the two solutions are identical, (2) the stress relations may be such as to allow more than one deformation to correspond to identical stress fields, so that $\bar{x}^{k}{ }_{, \alpha} \neq x^{k}{ }_{, \alpha}$ but $\bar{T}_{k}^{\alpha}=T_{k}^{\alpha},(3)$ the two solutions are distinct in such a way as to give the integrand both positive and negative values and zero mean value.

From (8.3) and (5.3) we see that the GCN condition contradicts (8.3) if $\bar{x}^{k}{ }_{\alpha}=G_{m}^{k} x^{m}, \alpha$ where $\boldsymbol{G}$ is positive-definite and symmetric. In order for such a condition to hold over a region, it is necessary and sufficient, since $\bar{x}^{k}, \alpha$ and $x^{m}, \alpha$ are both deformation gradients, that $\boldsymbol{G}$ also be a deformation gradient. Since $\boldsymbol{G}$ is symmetric, the displacement that gives rise to it must be derivable from a potential: $u_{k}=\Phi_{, k}$, where $\Phi$ is a scalar. Such deformations are called potential deformations $[1960,3, \S \S 36,38]$. We have thus established Uniqueness Theorem I: In an elastic material such that the GCN condition holds for all strains, a given mixed boundary-value problem cannot have two distinct solutions that differ from each other at each point by a pure potential deformation. This type of uniqueness is consistent with the general view of buckling, since the common and familiar buckled states certainly differ from the corresponding ground states by large and non-uniform local rotations. As a trivial corollary of the above theorem, or directly from the fact that $\mathrm{GCN} \Rightarrow \mathrm{IFS}$, we may infer that, for an elastic material as specified in the theorem, a given mixed boundary-value problem cannot be satisfied by more than one homogeneous strain.

To apply the above theorem we must be sure that the GCN condition is satisfied by all strains. We can weaken this requirement if we agree to consider only small strain superimposed upon a given, possibly large, strain, and if we replace $\mathrm{GCN}$ by $\mathrm{GCN}^{+}$. For if $\bar{x}^{m}{ }_{, \beta}-x_{,{ }_{, \beta}}^{m}$ is small, by (2.9), we see that

$$
\bar{T}_{k}^{\alpha}-T_{k}^{\alpha} \approx A_{k m}{ }^{\alpha \beta}\left(\bar{x}^{m}, \beta-x^{m},{ }_{\beta}\right),
$$

and if also $\bar{x}^{k}, \alpha=G_{m}^{k} x^{m}, \alpha$ where $\boldsymbol{G}$ is a symmetric tensor very nearly equal to 1 , then

$$
g_{k t} X^{\alpha}{ }_{, p}\left(\bar{x}^{k}, \alpha-x_{, \alpha}^{k}\right)=g_{k r}\left(G_{p}^{k}-\delta_{p}^{k}\right) \approx e_{p r}
$$

where $\boldsymbol{e}$ is an infinitesimal symmetric tensor. Thus by use of (2.9) we see that

$$
\left(\bar{T}_{k}^{\alpha}-T_{k}^{\alpha}\right)\left(\bar{x}^{k}, \alpha-x^{k}{ }_{\alpha}\right) \approx \frac{\varrho_{0}}{\varrho} B^{k m p q} \epsilon_{k m} e_{p q} .
$$

The foregoing calculation is formal and approximate. When we look back over it, we see that in fact any possible consequence of it may be obtained better from the rigorous analysis in $\S 6$. In particular, from (8.6) we might think that the GCN condition implies the inequality (6.10), but we know already that such is not the case, for only (6.9) can be proved rigorously. We have included the calculation leading to (8.6) only so as to make contact with the type of formal argument favored by many who cultivate mechanics today. Going back to the rigorous results in $\S 6$ and applying them directly to (8.3), we infer the following Uniqueness Theorem II: Let an elastic material bo subject to a particular strain such that the $\mathrm{GCN}^{+}$condition is satisfied everywerere in a certain region; 
then the mixed boundary-value problem for that region has at most one solution such that the difference between the resulting strain and the given strain is an infinitesimal pure strain. Note that this theorem does not yield a condition of stability. It remains possible that there may exist, corresponding to the same boundary conditions, other infinitesimal additional strains that are not pure. Thus, even for infinitesimal deformations (provided they be from a state of equilibrium subject to non-vanishing stress ${ }^{3}$ ), the $\mathrm{GCN}^{+}$condition is not so restrictive as to disallow infinitesimal rotation as a possible mechanism for instability.

Despite a formal similarity, the foregoing considerations are not to be confused with the result of HiLL ${ }^{4}$, who remarked that, as follows from (8.3) and (8.4), the condition

$$
A_{k m}{ }^{\alpha \beta} F_{\alpha}^{k} F_{\beta}^{m}>0
$$

for arbitrary $\boldsymbol{F}$ is sufficient for unqualified uniqueness of solution in the problem of small deformation superimposed upon an arbitrary deformation. HiLl put forward the inequality (8.7) as a possible criterion for stability. Unfortunately, it is self-contradictory. Indeed, if (8.7) holds, then the transformation from $\boldsymbol{F}$ to $\boldsymbol{T}=\boldsymbol{b}(\boldsymbol{F})$ is strongly convex:

$$
\operatorname{trace}\left\{[\boldsymbol{h}(\overline{\boldsymbol{F}})-\mathfrak{h}(\boldsymbol{F})]\left[\overline{\boldsymbol{F}}^{T}-\boldsymbol{F}^{T}\right]\right\}>0,
$$

for the fixed $\boldsymbol{F}$ corresponding to the given deformation and for arbitrary $\overline{\boldsymbol{F}}$ differing sufficiently little from $\boldsymbol{F}$. The inequality (8.8), however, is inconsistent with the principle of material indifference, which requires that ${ }^{5}$

$$
\mathfrak{h}(\boldsymbol{Q} \overline{\boldsymbol{F}})=\boldsymbol{Q} \mathfrak{h}(\overline{\boldsymbol{F}})
$$

for any $\overline{\boldsymbol{F}}$ and any orthogonal $\boldsymbol{Q}$. To show that (8.8) and (8.9) are inconsistent, consider an infinitesimal rotation $\boldsymbol{Q}$ of a material having a natural state. Set $\boldsymbol{F}=\mathbf{1}$, so that $\mathfrak{h}(\boldsymbol{F})=\mathfrak{h}(\mathbf{1})=\mathbf{0}$, and take $\overline{\boldsymbol{F}}=\boldsymbol{Q}$. Then by (8.9) we have

$$
\begin{aligned}
{[\mathfrak{h}(\overline{\boldsymbol{F}})-\mathfrak{b}(\boldsymbol{F})]\left[\overline{\boldsymbol{F}}^{T}-\boldsymbol{F}^{T}\right] } & =\mathfrak{h}(\boldsymbol{Q})\left(Q^{T}-1\right), \\
& =\boldsymbol{Q} \mathfrak{h}(\mathbf{1})\left(\boldsymbol{Q}^{T}-1\right), \\
& =\mathbf{0} .
\end{aligned}
$$

Hence (8.8) can never be satisfied by all pairs $\boldsymbol{F}, \overline{\boldsymbol{F}}$, or even by all pairs $\boldsymbol{F}, 1$ as appropriate to the classical linearized theory. Consequently, HiLL's sufficient condition for uniqueness is empty.

${ }^{3}$ This qualification is explained as follows. To derive (8.6) we have assumed that $\bar{x}^{k}, \alpha=G_{m}^{k} x^{m}, \alpha$, where $\boldsymbol{G}$ is symmetric. This restriction, however, is sufficient, not necessary, for the truth of (8.6). From formulae given by TrUesdell [1961,6, Eq. (6.10)] it is clear that while (8.6) does not hold in general for arbitrary $\bar{x}^{k}, \alpha$, it does so hold in the case when $T_{k}^{\alpha}=0$, i.e., for infinitesimal strain from the natural state. Thus, as follows more directly from (5.9), the GCN condition suffices to insure uniqueness of solution in the mixed boundary-value problem of the linearized theory, without qualification.

$4[1957,2, \S \S 2,3]$. The more elaborate analysis of GREEN \& ADKINS [1960, 1, §9.3] seems to fall back to the same observation. The remarks of these three authors might give the unwary reader the notion, wholly false, that the uniqueness theorem of ERICKSEN \& ToupIN [1956, 1, §7] amounts to no more than this, although a later paper of HILL $[1962,5, \S 4]$ clears the point. HILL's remark that there is no reason to expect uniqueness to fail when his condition (8.7) fails is borne out by our proof that his condition can never hold.

- Noll [1955, Eq. (15.2)]. Those who prefer a more formal argument may derive (8.9) from (2.1). 
The reader may ask, when KIRCHHOFF's argument goes through perfectly in the linearized theory, why does HiLL's, so closely modelled upon it, fail for the finite theory? The answer is that KIRCHHOFF began with an identity from which the rotation had already been eliminated; hence his result satisfies, trivially, the invariance requirements of the linearized theory. In HILI's starting point (8.2), or (8.3), the rotation is very much in evidence, and the condition (8.8) distegards the invariance of elastic response under rigid-body motion. Despite a formal similarity, Hill's argument and KiRchнofF's are not conceptually parallel. A true extension of KIRCHHOFF's theorem is given by our Uniqueness Theorem II.

It should be remarked that most of HILL's work refers to the weaker inequality

$$
\int_{\dot{m}_{0}} A_{k m}^{\alpha \beta} u^{k}{ }_{\alpha \alpha} u^{m}, \beta d V_{0}>0
$$

for all not identically vanishing vectors $\boldsymbol{u}$ that vanish on $\partial \mathscr{S}_{0}$. The condition $(8.10 \mathrm{~A})$ is indeed sufficient for uniqueness, since the difference $\boldsymbol{u}$ of any two solutions to the same displacement boundary-value problem for infinitesimal strain superimposed upon a given strain must' satisfy the condition

$$
\int_{\mathscr{B}_{0}} A_{k m}{ }^{\alpha \beta} u^{k}, \alpha u^{m}, \beta d V_{0}=0,
$$

as follows from (8.4) and (8.3). Between (8.10A) and (8.10B) there is a plain contradiction. As remarked by Hill in a later paper ${ }^{6}$, a uniqueness theorem in the usual sense of the term can be inferred by making further assumptions:

1. The given strain is homogeneous.

2. The S-E condition holds at each point of $\mathscr{B}_{0}$.

It can then be proved that (8.10A) does hold. The resulting assertion of uniqueness is included as a special case in our Uniqueness Theorem III, below.

Uniqueness Theorem II is not to be confused with the uniqueness theorem that results from imposing the S-E condition. From (2.9) it is easy to show that (7.1) is equivalent to

$$
A_{k m}{ }^{\alpha \beta} l^{k} l^{m} \cdot \lambda_{\alpha} \lambda_{\beta}>0 .
$$

Now the differential equations satisfied by $u^{k}$, the small additional displacement from the given deformed state, are of the form

$$
\left(A_{k m}^{\alpha \beta} u^{m}, \beta\right)_{; \alpha}+\varrho_{0} b_{k}=0,
$$

where the coefficients $A_{k m}{ }^{\alpha \beta}$ are known functions of position, and where the body force $\varrho_{0} b_{k}$ is likewise prescribed. From a known property of strongly elliptic differential systems we may read off ${ }^{7}$ Uniqueness Theorem III: Let

B $[1962,5, \S 4($ ii) $]$.

7 For hyperelastic materials, ERICKSEN \& ToupIN [1956, 1, Th. 1] obtained this result by direct proof. An elastic material is hyperclastic if and only if

$$
A_{k m}^{\alpha \beta}=A_{m k}^{\beta \alpha} \text {. }
$$

This condition renders the operator $A_{k} m^{\alpha \beta}$ self-adjoint and hence has numerous important consequences, but it is not needed for the general theorems of existence and uniqueness proved by BRowDER $[1954,2]$, MORREY $[1954,3]$, and others.

Perhaps we should remark that these theorems cannot be applied in any obvious way to the general displacement boundary-value problem of elasticity, since they do not allow $A_{k m}{ }^{\alpha \beta}$ to depend upon $\imath^{q} ; \gamma$. Researches on the theory of partial dif- 
an elastic material be subject to a particular strain such that the $S-E$ condition is satisfied everywhere in a certain region; then the displacement boundary-value problem for that region has at most one solution such that the difference between the resulting strain and the given strain is infinitesimal. Unlike Uniqueness Theorem II, this theorem is connected with criteria of stability, as shown by the researches of HADAMARD and DuHEM, to which we referred at the beginning of this paper, and by the various known results concerning wave motions ${ }^{8}$. It cannot be extended to the stress boundary-value problem, as shown by results summarized in Footnote 3 to $\$ 1$.

Be it noted that existence of a stored-energy function is not presumed for any of the three uniqueness theorems just stated and proved.

\section{General conclusions}

In case the logical threads tracked in the foregoing pages may seem intricate, here we select for repetition a few of the conclusions we deem most important.

Two classes of conditions have been studied. One consists in the condition of strong ellipticity (S-E); the other, in various closely related but not equivalent forms of the condition of Coleman \& Noll (C-N). Neither class includes the other, though they have a domain of common validity. In particular, for the classical linearized theory all the conditions considered in this paper follow as consequences of the classical inequalities (1). Our study concerns mainly the second class.

As the most general form of condition (GCN) of the type first suggested by Coleman \& NolL, we propose the statement that the transformation from the deformation gradient $x^{k}, \alpha$ to the Piola-Kirchhoff stress $T_{k}^{\alpha}$ be a convex transformation if the local rotation is kept fixed. It follows that certain combinations of elasticities must yield a quadratic form in six variables that is positive-definite except on a nowhere dense set. The stronger requirement obtained by disallowing altogether such an exceptional set we call the $\mathrm{GCN}^{+}$condition. For an isotropic material, whether or not it have a stored-energy function, the $\mathrm{GCN}^{+}$ condition suffices to insure that:

ferential equations do not seem yet to approach the level of generality necessary for study of classical finite elasticity as a branch of analysis. Likewise the specific work of STOPPELLI $[1954,4]$ does not cast any light on our problem since, referring only to a sequence of applied loads approaching zero, it finds that a sufficient condition for existence and uniqueness of solutions to a corresponding sequence of stress boundary-value problems is the existence and uniqueness of solution to the resulting linearized problem.

According to ZoRski [1962,6, Introd.] "... the condition of strong ellipticity ensures both the uniqueness and existence of the first boundary value problem (displacements described on the boundary); these results can easily be extended to include the second and the third (mixed) boundary value problem." However, this assertion cannot be true, since, as was mentioned in footnote 3 to $\S 1$, strong ellipticity does not suffice for uniqueness of solution to the stress boundary-value problem even in the linearized theory.

8 Toupin \& Bernstein [1961, 5], Hayes \& Riviln [1961, 3], but note especially the conjecture of Truesdell [1961,6, end of $\S 5]$. 
1. If there is a natural state, then, in mean, pressure is needed to effect compression and tension to effect expansion from that state (P-C).

2. The force-stretch relations are uniquely invertible and in fact have a positive Jacobian (C \& IFS ${ }^{+}$.

3. The curve of principal force $T_{a}$ against the corresponding principal stretch $v_{a}$, when the other principal stretches are kept constant, slopes upward $\left(\mathrm{T}-\mathrm{E}^{+}\right)$.

4. The curve of principal stretch $v_{a}$ against the corresponding principal force $T_{a}$, when the other prinicpal forces are kept constant, slopes upward $\left(\mathrm{E}-\mathrm{T}^{+}\right)$.

5. The greater principal force corresponds always to the greater principal stretch $(\mathrm{O}-\mathrm{F})$.

6. If, starting from a given state of strain, possibly severe, two different small defarmations corresponding to the same mixed boundary values are possible, then they cannot differ by a pure strain (Uniqueness Theorem II).

The converse proposition is of two parts. In order for the $\mathrm{GCN}^{+}$condition to hold, it suffices that $A_{\Gamma}>0$ and that the Jacobian matrix of the transformation from principal stretches to principal forces be positive-definite. The former condition, while indeed purely statical, we have been unable to interpret in general, although we have found simple inequalities sufficient to ensure it. The latter, in the case when a stored-energy function exists, we have shown to be equivalent to the assertions numbered 2,3 , and 4 .

The results we have presented seem to us to add weight in favor of conditions of Coleman \& Noll's type as a material requirement, a requirement, that is, to be imposed on the form of the stress-strain relations, for all strains of all elastic materials, or at least, let us say, as a hypothesis to be used when normal behavior is to be described by the mathematical model. The general theory of waves, supplemented by Uniqueness Theorem III, seems to us to show, on the other hand, that the condition of strong ellipticity is not a material condition but is rather a criterion of stability for a particular strain of a particular material.

We are grateful to Messrs. Noll and BRAGG for detecting errors in an earlier version of this paper and for informing us of various complementary results. The work of TRUESDELI. was supported by a grant from the U.S. National Science Foundation.

\section{References}

[1888] Thomson, W.: On the reflection and refraction of light. Phil. Mag. (5) 26, 414-425. Reprinted in part as $\S \S 107-111$ of Baltimore Lectures on Molecular Dynamics and the Wave Theory of Light. London: Clay and Sons 1904.

[1903] Hadamard, J.: Leçons sur la Propagation des Ondes. Paris: Hermann.

[1905] Duнem, P.: Recherches sur l'élasticité, Troisième partie.' la stabilité des milieux élastiques. Ann. Ecole Norm. (3) 22, 143-217.

[1907] Boggro, T.: Nuova risoluzione di un problema fondamentale della teoria dell'elasticità. Rend. accad. Lincei (5) 16, 248-255.

[1949] Signorini, A.: Trasformazioni termoelastiche finite. Memoria 2a. Annali di Mat. Pur. Appl. (4) 30, 1-72.

[1950] FicherA, G.: Sull'esistenza e sul calcolo delle soluzioni dei problemi al contorno relativi all'equilibrio di un corpo elastico. Ann. Sc. Norm. Pisa (3) 4, 35-99. 
[1952] Truesdell, C.: The mechanical foundations of elasticity and fluid dynamics. J. Rational Mech. Anal. 1, 125-300. To be reprinted shortly, in corrected form, in the International Science Review Series, Gordon and Breach, New-York.

[1953, 1] ERICKSEN, J. L.: On the propagation of waves in isotropic incompressible elastic materials. J. Rational Mech. Anal. 2, 329-337.

$[1953,2]$ Mișıcu, M.: Echilibrul mediilor continue cu deformâri mari. Studii şi cerc. mec. metal. 4, 31-53.

$[1954,1]$ BAKER, M., \& J. L. ERICKSEN : Inequalities restricting the form of the stress deformation relations for isotropic elastic solids and Reiner-Rivlin fluids. J. Wash. Acad. Sci. 44, 33-35.

$[1954,2]$ Browder, F. E.: Strongly elliptic systems of differential equations. Contrib. Theory Partial Diff. Eqns., Annals Math. Studies No. 33, 15-51.

[1954, 3] Morrey, C. B.: Second order elliptic systems of differential equations. Contrib. Theory Partial Diff. Eqns., Annals Math. Studies No. 33, 101-159.

[1954, 4] Stoppelli, F.: Un teorema di esistenza e di unicità relativo alle equazioni dell'elastostatica isoterma per deformazioni finite. Ricerche mat. 3, $247-267$.

[1955] Noll, W.: On the continuity of the solid and fluid states. J. Rational Mech. Anal, 4, 3-81.

[1956, 1] ERICKSEN, J. L., \& R. A. TOUPIN: Implication of Hadamard's condition for elastic stability with respect to uniqueness theorems. Canad. J. Math. $8,432-436$.

[1956, 2] Truesdell, C.: Das ungelöste Hauptproblem der endlichen Elastizitätstheorie. Z. angew. Math. Mech. 36, 97-103.

$[1957,1]$ Barta, J.: On the non-linear elasticity law. Acta Tech. Acad. Sci. Hungaricae 8, 55-65.

$[1957,2]$ ERICKSEN, J. L.: On the Dirichlet problem for linear differential equations. Proc. Amer. Math. Soc. 8, 521-522.

[1957, 3] HiLl, R.: On uniqueness and stability in the theory of finite elastic strain. J. Mech. Phys. Solids 5, 229-241.

[1959] Colfman, B. D., \& W. Noll: On the thermostatics of continuous media. Arch. Rational Mech. Anal. 4, 97-128.

[1960, 1] Green, A. E., \& J. E. Adkins: Large Elastic Deformations and Non-linear Continuum Mechanics. Oxford: Clarendon Press.

$[1960,2]$ Gurtin, M. E., \& E. SternberG: On the first boundary-value problem of linear elastostatics. Arch. Rational Mech. Anal. 6, 177-187.

$[1960,3]$ Truesdell, C., \& R. Toupin: The Classical Field Theories. Flügge's Encyclopedia of.Physics 3, 226-793. Berlin-Göttingen-Heidelberg: Springer.

$[1961,1]$ Brdmble, J. H., \& L. E. PAYxe: An analogue of the spherical harmonics for the equations of elasticity. J. Math. Phys. 15, 163-171.

$[1961,2]$ Gurtix, M. E., \& E. SternberG: A note on uniqueness in classical elastodynamics. Q. Appl. Math. 19, 169-171 (1961/2).,

[1961,3] Hay'es, M., \& R. S. Rivlin: Propagation of plane waves in an isotropic elastic material subject to pure homogeneous deformation. Arch. Rational Mech. Anal. 8, 15-22.

[1961,4] Hill, R.: Uniqueness in general boundary-value problems for elastic or inelastic solids. J. Mech. Phys. Solids 9, 114-130.

$[1961,5]$ Toupre, R. A., \& B. Bernstein: Sound waves in perfectly elastic materials. Acoustoelastic effect. J. Acoust. Soc. Amer. 33, 216-225.

[1961, 6] Truesdel, C.: General and exact theory of waves in finite elastic strain. Arch. Rational Mech. Anal. 8, 263-296.

1962, 1] Bersstein, B., \& R. A. Toupin: Some properties of the Hessian matrix of a strictly convex function. J. Reine Angew. Math. 210, 65-72.

[1962, 2] BRAmble, J. H., \& L. E. PAYNE: Some uniqueness theorems in the theory of elasticity. Arch. Rational Mech. Anal. 9, 319-328. 
[1962, 3] Bramble, J. H., \& L. E. Payne: Proc. 4th U.S. Congr. Applied Mech. forthcoming.

$[1962,4]$ Coleman, B. D.: Mechanical and thermodynamical admissibility of stressstrain functions. Arch. Rational Mech. Anal. 9, 172-186.

$[1962,5]$ Hrll, R.: Acceleration waves in solids. J. Mech. Phys. Solids 10, 1-16.

$[1962,6]$ ZoRsKI, H.: On the equations describing small deformations superimposed on finite deformations. Proc. Int. Sympos. Second-order Effects Haifa, forthcoming.

[1963] BRAGG, L. E., \& B. D. Coleman: On strain energy functions for isotropic elastic materials. In preparation.

The Johns Hopkins University Baltimore, Maryland and

Watson Research Center

Yorktown Heights, New York 